\title{
Multiplicity of solutions for laminar, fully-developed natural convection in inclined, parallel-plate channels
}

\author{
Marzio Piller \\ Dipartimento di Ingegneria Civile e Architettura \\ Università degli Studi di Trieste \\ via A. Valerio 6/1 - 34127 Trieste, Italy \\ Sergio Polidoro \\ Dipartimento Scienze Fisiche, Informatiche, Matematiche \\ Università degli Studi di Modena e Reggio Emilia \\ Via Campi, 213/A - 41125 Modena, Italy \\ Enrico Stalio* \\ Dipartimento di Ingegneria "Enzo Ferrari" \\ Università degli Studi di Modena e Reggio Emilia \\ Strada Vignolese, 905/B - 41125 Modena, Italy
}

\begin{abstract}
Natural convection in inclined channels is a rather common flow configuration: it occurs in solar energy systems, ventilated roofs as well as in many industrial applications and chemical processes. Analytical solutions for laminar, fully-developed natural convection in inclined parallel-plate channels are presented in this paper. The Boussinesq approximation is applied and viscous energy dissipation is neglected. One specific thermal configuration is addressed, where one wall is perfectly insulated and a constant, uniform heat flux is released to the fluid from the other wall. The resulting set of governing equations is non-linear, as the mean velocity is not assigned a priori but determined as part of the solution.

Depending on the channel inclination angle and on the imposed heat flux conditions, either no solution, one solution, multiple or infinite solutions
\end{abstract}

\footnotetext{
${ }^{*}$ Corresponding Author

Email address: enrico.stalio@unimore.it (Enrico Stalio)
} 
exist. Under restrictive assumptions velocity profiles are self-similar with respect to the channel inclination, while the temperature profile is independent of the inclination. The two-dimensional, hydraulically- and thermallydeveloping natural convection channel flow is simulated numerically for some combinations of channel inclination angle and heating intensity to identify the most physical between the many solutions.

Key words: Natural convection; Inclined channel; Analytical solution; Multiple solutions; Fully developed; Isoflux conditions 


\section{Nomenclature}

\section{Subscripts}

$\pi / 2$ vertical channel

* scale value for the dimensionless form

ref conditions at $x=0, y=0$, taken as reference conditions

$w$ wall conditions

\section{Greek letters}

$\alpha$ solution parameter for the $U_{m}>0$ case, equation (36)

$\beta_{\text {ref }}$ thermal expansion coefficient

$\kappa_{\text {ref }}$ thermal diffusivity at reference conditions, $\kappa_{\text {ref }}=\lambda_{\text {ref }} /\left(\rho_{\text {ref }} c\right)$

$\lambda$ thermal conductivity of the fluid

$\mu$ viscosity

$\nu$ kinematic viscosity

$\partial_{x}$ partial derivative in $x$

$\rho$ density

$\sigma$ solution parameter for the $U_{m}<0$ case, equation (38)

$\Theta$ non dimensional, normalized temperature field

$\varphi$ channel inclination angle

$\vartheta$ normalized temperature, see (5)

\section{Latin letters}

$\overline{\widetilde{p}} y$-averaged modified pressure field

$\bar{p} y$-averaged pressure field

$\Phi_{\Theta}$ enthalpy flux, equation (44)

$\mathrm{Ra}_{*}$ Rayleigh number based on the temperature scale $T_{*}$, see equation (22)

$\boldsymbol{g}$ acceleration due to gravity

$\widehat{P}$ dimensionless pressure field, see equation 19

$\widetilde{p}$ pressure field clear of the hydrostatic contribution induced by temperature, see eq. (8)

$A$ solution parameter, see equation (34); in the present study $A \neq 0$

$c_{p}, c_{v}$ specific heat at constant pressure, volume

$E_{\Theta}$ averaged square temperature, see equation (45)

$E_{U}$ momentum flux, equation (43)

$F_{\Theta} x$-component of the buoyancy force induced by temperature differences in $y$-direction

$F_{p}$ pressure term, see equation (49) 
$F_{v}$ viscous force term

$H$ height of the channel, reference length

$m$ heating ratio, see equation (25)

$p$ pressure field

$p_{*}$ scale for the pressure, $(20)$

$p_{m}$ modified pressure, clear of the hydrostatic contribution $p_{m}=p-p_{s}[3]$

$p_{s}$ static pressure, gives rise to a pressure gradient in balance with the force of gravity [3]

$q_{0}^{\prime \prime}$ uniform heat flux imposed at the lower wall

$q_{H}^{\prime \prime}$ uniform heat flux imposed at the upper wall

$T$ temperature

$T_{*}$ temperature scale, equation (20)

$T_{b}$ bulk temperature $T_{b}=H^{-1} u_{m}^{-1} \int_{0}^{H} u T \mathrm{~d} y$

$T_{w}$ (x) local wall temperature

$U$ non dimensional, streamwise component of the velocity

$u$ streamwise component of the velocity

$U_{*}$ velocity scale, equation $(20)$

$u_{m}$ mean velocity $u_{m}=H^{-1} \int_{0}^{H} u \mathrm{~d} y$

$v_{g}$ buoyancy-related velocity scale, see equation (1)

$X, Y$ non dimensional coordinates

$x, y$ Cartesian coordinates

Ec Eckert number, see equation (1)

\section{Superscripts}

$(n)$ negative $U_{m}$ solution

$(p)$ positive $U_{m}$ solution

I, II, II', III' first to fourth-order derivatives with respect to $y$ 


\section{Introduction}

Free convection between parallel plates, at low Rayleigh numbers, is of relevant importance in several practical applications, as air conditioning, solar energy systems, ventilated roofs, chemical vapor deposition, cooling of electronic equipment [2], heating of buildings via Trombe walls [1, 8], and the cooling of nuclear fuel elements, to mention a few. While a vast literature is available for vertical channels, only few contributions tackle free convection in inclined channels.

The problem of fully-developed, laminar natural convection in vertical channels with constant, but not necessarily equal, wall temperatures is addressed by Ostrach [14]. The resulting sequence of fourth-order, non-linear, ordinary differential equations is solved by a method of successive approximations, where the frictional heating term is taken from the most recently available solution.

The stability of natural convection flow between vertical, parallel plates kept at fixed, different temperatures is addressed by Vest and Arpaci [18], by Gershuni and Zhukovitskii [6] and by Bergholz [4]. After Vest and Arpaci both the conduction and boundary-layer regimes are unstable with respect to stationary disturbances in the form of multicellular secondary flows. Experimental measurements are reported, confirming the theoretical predictions.

Gershuni and Zhukovitskii [6] investigate the stability of natural convection flow between vertical plates, with a longitudinal temperature gradient and a fixed temperature difference between the plates. An opposing temperature gradient has a destabilizing effect for this flow configuration. The instability effect can be either hydrodynamic, due to the increased base velocity caused by the longitudinal temperature gradient, or convective, due directly to the destabilizing effect of the adverse temperature gradient.

Bergholz [4] studies the instability of steady free convection between vertical surfaces kept at different temperatures. The linear stability theory is used to obtain the critical Grashof and Rayleigh numbers, for Prandtl numbers ranging from 0.73 to 1000 and for the limiting case of infinite Prandtl

number. The modes of instability depend on the Prandtl number and are investigated by numerical simulation.

Experimental and numerical investigations of the free convection in vertical channels are as well reported in the literature. Sparrow and Azevedo [17] investigate both experimentally and numerically the natural convection in open-ended vertical channels bounded by an isothermal and an unheated 
wall. The experiments are performed in water, with a nominal Prandtl number of 5 . The numerical model takes into account thermal conduction within the unheated wall. It is found that the flat-plate results do not form the upper bound for the heat transfer rates for the channel. The sensitivity of the Nusselt number to the inter-plate spacing and to the Rayleigh number is investigated.

Webb and Hill [19] measure local heat transfer for the laminar, natural convective flow of air between vertical parallel plates heated asymmetrically. A uniform heat flux is imposed along one heated wall, while the opposing wall of the channel is thermally insulated. Correlations are presented for the local Nusselt number as a function of local Grashof number along the channel. The dependence of both the average Nusselt number and the maximum heated wall temperature on the modified Rayleigh number is also explored. Results are in good agreement with previous analytical and experimental investigations.

Within the few contributions that consider free convection close to inclined walls, Hart [7] carries out a theoretical and experimental study of free convection in water, within a differentially heated, inclined rectangular box of small width-to-length aspect ratio. A basic state which exists for low Rayleigh numbers becomes unstable as this parameter is increased. The types of instability and manner in which the motions become turbulent depend crucially on the inclination angle. Agreement between the stability theory and the experiments is claimed to be generally quite good over the whole range of the inclination angle.

Lavine [10] presents an exact solution for fully developed, laminar mixed convection between inclined parallel plates with uniform wall heat flux released from both walls into the channel. The flow is upward, so that natural convection aids the forced flow. Different flow regimes are identified, some of them are characterized by the presence of velocity minima or flow reversals. Lavine [11] derives also an analytical solution for the fully-developed, opposing, laminar mixed convection between inclined parallel plates. The channel is uniformly heated from both walls and the external pressure gradient causes a descending flow. The original governing equations are combined to yield a fourth-order, linear, ordinary differential equation (ODE) for the velocity field, with a similar technique as the one proposed in the present investigation. It is noticed that the pressure field does not vary linearly with the streamwise coordinate while a quadratic streamwise variation of the temperature field is obtained. The onset of different flow configurations is observed, 
some of them characterized by flow reversal. The forced convection flow configuration can be derived as a limiting case of the proposed mixed convection analysis. Conversely the free convection case can not be recovered using a similar limiting procedure [11].

A linear stability analysis for mixed and natural convection between infinite, parallel plates, arbitrarily inclined with respect to gravity is also performed by Lavine [12]. Fixed heat flux boundary conditions are enforced at the walls and a linear streamwise variation of the mean temperature is assumed. In the case of adverse temperature gradient it is found that the flow is unstable for any arbitrary base velocity and temperature fields and for each channel inclination.

Piller and Stalio [15] obtain numerical results of laminar fully developed free convection in an inclined channel partly filled with metal foam. A uniform heat flux is released from the wall on which the porous insert is mounted, while the solid wall in contact with the fluid is perfectly insulated. The Boussinesq approximation is used, while viscous energy dissipation is neglected. One of the main findings is that for negligible non-Darcian effects and thermal dispersion, the Nusselt number of the composite system in free convection regime is independent of the channel inclination.

The present work investigates analytically the laminar free convection between two infinite parallel plates, variably inclined with respect to the direction of gravity. A given heat flux is released form one wall, while the other is perfectly insulated. In spite of some simplifications, the assumed thermal boundary conditions yield a set of non-linear ordinary differential equations. The non-linearity enters the problem in the form of the mean flow velocity, which is unknown a priori and is determined by enforcing global energy conservation as an additional condition.

Dimensionless solutions depend only on parameter $A$, accounting for the channel inclination and the Rayleigh number $\mathrm{Ra}_{*}$, while they are independent of the Prandtl number. Different ranges of the parameter $A$ are characterized by either no solutions, a single solution, a finite or infinite sequence of solutions. Numerical simulations of the steady, hydrodynamically and thermally developing flow confirm the analytical results and allow to single out a physical solution in cases where the fully-developed model provides multiple solutions. A major result of the present investigation is the identification of limits on relevant dimensionless groups, within which the non-dimensional solution is self-similar with respect to the channel inclination, see also Piller and Stalio [15]. 


\section{Mathematical model}

\subsection{Physical assumptions and resulting equations}

The flow configuration considered in this research is sketched in figure 1 . Isoflux boundary conditions are enforced at both walls.

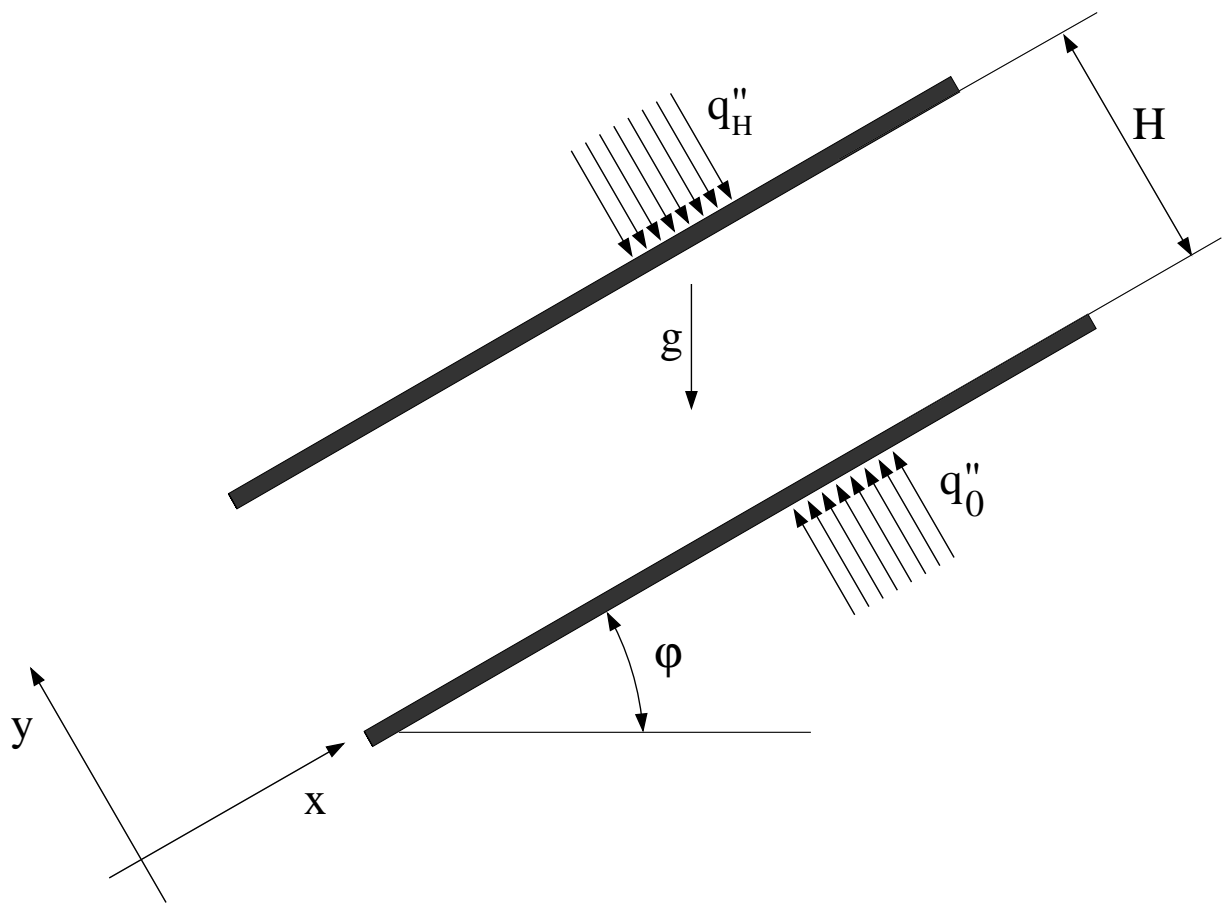

Figure 1: Two-dimensional representation of the inclined channel.

A fully developed, laminar natural convection flow of a Newtonian fluid with constant thermophysical properties is considered. The Boussinesq approximation is assumed to account for density variations. Viscous energy dissipation is neglected which implies assuming Ec $\ll 1$, as discussed by Ostrach [14]. Ec denotes the Eckert number based on the buoyancy-related velocity scale $v_{g}$ :

$$
v_{g} \equiv \sqrt{\beta_{\mathrm{ref}} g T_{*} H} \quad \mathrm{Ec} \equiv \frac{v_{g}^{2}}{c_{p} T_{*}}
$$

where $T_{*}$ is a reference temperature difference and $H$ a reference length. 
Under the aforementioned assumptions, the momentum and energy conservation equations for the considered flow and heat transfer configuration can be cast as

$$
\begin{gathered}
0=\beta_{\text {ref }}\left(T-T_{\text {ref }}\right) g \sin (\varphi)+\nu_{\text {ref }} \frac{\mathrm{d}^{2} u}{\mathrm{~d} y^{2}}-\frac{1}{\rho_{\text {ref }}} \frac{\partial p_{m}}{\partial x} \\
0=\beta_{\text {ref }}\left(T-T_{\text {ref }}\right) g \cos (\varphi)-\frac{1}{\rho_{\text {ref }}} \frac{\partial p_{m}}{\partial y} \\
u \frac{\partial T}{\partial x}=\kappa_{\text {ref }}\left(\frac{\partial^{2} T}{\partial x^{2}}+\frac{\partial^{2} T}{\partial y^{2}}\right)
\end{gathered}
$$

where the modified pressure $p_{m}$ is defined as [3]:

$$
p_{m} \equiv p-\rho_{\text {ref }} \boldsymbol{g} \cdot \boldsymbol{x}
$$

The modified pressure $p_{m}$ is rid of the hydrostatic contribution.

The isoflux thermal boundary conditions on the solid walls correspond to:

$$
\begin{aligned}
& -\left.\lambda \frac{\partial T}{\partial y}\right|_{y=0}=q_{0}^{\prime \prime} \\
& \left.\lambda \frac{\partial T}{\partial y}\right|_{y=H}=q_{H}^{\prime \prime}
\end{aligned}
$$

The fully-developed assumption [9] allows to conclude that the temperature field varies linearly with $x$ and takes the general form

$$
T(x, y)=T_{w}(x)+\vartheta(y)
$$

Equation (5) can also be used to define the temperature difference $\vartheta(y)$. Conditions at $x=0, y=0$ are selected as reference conditions $T_{\text {ref }} \equiv T(0,0)$.

A global energy balance yields to the axial variation of the bulk temperature

$$
\frac{\mathrm{d} T_{b}}{\mathrm{~d} x}=\frac{q_{H}^{\prime \prime}+q_{0}^{\prime \prime}}{\rho_{\mathrm{ref}} c_{p} u_{m} H}
$$

In view of the considerations above, the momentum and energy equations can be recast as

$$
0=\beta_{\text {ref }} g \sin (\varphi) \vartheta(y)+\beta_{\text {ref }} g \sin (\varphi) \frac{\mathrm{d} T_{b}}{\mathrm{~d} x} x-\frac{1}{\rho_{\text {ref }}} \frac{\partial p_{m}}{\partial x}+\nu_{\text {ref }} \frac{\mathrm{d}^{2} u}{\mathrm{~d} y^{2}}
$$




$$
\begin{gathered}
0=\beta_{\text {ref }} g \cos (\varphi) \vartheta(y)+\beta_{\text {ref }} g \cos (\varphi) \frac{\mathrm{d} T_{b}}{\mathrm{~d} x} x-\frac{1}{\rho_{\text {ref }}} \frac{\partial p_{m}}{\partial y} \\
u \frac{\mathrm{d} T_{b}}{\mathrm{~d} x}=\kappa_{\mathrm{ref}} \frac{\mathrm{d}^{2} \vartheta}{\mathrm{d} y^{2}}
\end{gathered}
$$

In order to further simplify equation (7a) it is convenient to introduce a second modified pressure $\widetilde{p}$, accounting for the temperature variation in the axial direction

$$
\widetilde{p} \equiv p_{m}-\rho_{\text {ref }} g \sin (\varphi) \beta_{\text {ref }} \frac{\mathrm{d} T_{b}}{\mathrm{~d} x} \frac{x^{2}}{2}
$$

leading to the following form of the momentum equations

$$
\begin{gathered}
0=\beta_{\text {ref }} g \sin (\varphi) \vartheta-\frac{1}{\rho_{\text {ref }}} \frac{\partial \widetilde{p}}{\partial x}+\nu_{\text {ref }} \frac{\mathrm{d}^{2} u}{\mathrm{~d} y^{2}} \\
0=\beta_{\text {ref }} g \cos (\varphi) \vartheta+\beta_{\text {ref }} g \cos (\varphi) \frac{\mathrm{d} T_{b}}{\mathrm{~d} x} x-\frac{1}{\rho_{\text {ref }}} \frac{\partial \widetilde{p}}{\partial y}
\end{gathered}
$$

Taking the $x$-derivative of both $(9 \mathrm{a})$ and $(9 \mathrm{~b})$ reveals that $\partial_{x} \tilde{p}$ is independent of $x$ and linear in $y$. It can be concluded that the streamwise pressure gradient has the following functional form:

$$
\frac{\partial \widetilde{p}}{\partial x}=b_{1} y+b_{0}
$$

where

$$
b_{1}=\rho_{\text {ref }} g \beta_{\text {ref }} \cos (\varphi) \frac{\mathrm{d} T_{b}}{\mathrm{~d} x}
$$

and $b_{0}$ is a constant to be determined.

Integration in the wall normal direction and subsequent derivation in $x$ of equation (8) gives

$$
\frac{\mathrm{d} \bar{p}}{\mathrm{~d} x}=\frac{\mathrm{d} \overline{\widetilde{p}}}{\mathrm{~d} x}-\rho_{\text {ref }} g \sin (\varphi)\left(1-\beta_{\mathrm{ref}} \frac{\mathrm{d} T_{b}}{\mathrm{~d} x} x\right)
$$

where

$$
\frac{\mathrm{d} \bar{p}}{\mathrm{~d} x}=\frac{1}{H} \int_{0}^{H} \frac{\partial p}{\partial x} \mathrm{~d} y
$$


It is now shown that the $x$-derivative of the $y$-averaged modified pressure $\overline{\widetilde{p}}$ must be constant: assuming $\mathrm{d} \overline{\widetilde{p}} / \mathrm{d} x \neq 0$, the following momentum equation is derived from equations (9a), (10) and (11)

$$
0=\beta_{\text {ref }} g\left[\vartheta \sin (\varphi)-\left(y-\frac{H}{2}\right) \frac{\mathrm{d} T_{b}}{\mathrm{~d} x} \cos (\varphi)\right]-\frac{1}{\rho_{\text {ref }}} \frac{\mathrm{d} \overline{\tilde{p}}}{\mathrm{~d} x}+\nu_{\mathrm{ref}} \frac{\mathrm{d}^{2} u}{\mathrm{~d} y^{2}}
$$

where $\mathrm{d} \overline{\vec{p}} / \mathrm{d} x$ is a constant that can be assigned independently of the heating mode. As the interest is in natural convection solutions, i.e. velocity fields that vanish for isothermal flow, equation (14) can only be satisfied by

$$
\frac{\mathrm{d} \overline{\tilde{p}}}{\mathrm{~d} x}=0
$$

Equation (15) can be used to determine the constant $b_{0}$ in (10)

$$
b_{0}=-\rho_{\text {ref }} g \beta_{\text {ref }} \cos (\varphi) \frac{\mathrm{d} T_{b}}{\mathrm{~d} x} \frac{H}{2}
$$

The axial derivative of the second modified pressure is therefore expressed as

$$
\frac{\partial \widetilde{p}}{\partial x}=\rho_{\text {ref }} g \beta_{\text {ref }} \cos (\varphi) \frac{\mathrm{d} T_{b}}{\mathrm{~d} x}\left(y-\frac{H}{2}\right)
$$

Equations (9a) and (7c) allow to solve for $u$ and $\vartheta$. Then the pressure distribution can be computed from $(9 \mathrm{~b})$. The resulting differential problem for $u$ and $\vartheta$ is given by:

$$
\begin{gathered}
\nu_{\text {ref }} \frac{\mathrm{d}^{2} u}{\mathrm{~d} y^{2}}+\beta_{\text {ref }} g \sin (\varphi) \vartheta-g \beta_{\text {ref }} \cos (\varphi) \frac{\mathrm{d} T_{b}}{\mathrm{~d} x}\left(y-\frac{H}{2}\right)=0 \\
\lambda_{\text {ref }} \frac{\mathrm{d}^{2} \vartheta}{\mathrm{d} y^{2}}-\frac{q_{H}^{\prime \prime}+q_{0}^{\prime \prime}}{H} \frac{u}{u_{m}}=0 \\
u(0)=0 ; \quad u(H)=0 \\
q_{0}^{\prime \prime}=-\left.\lambda_{\text {ref }} \frac{\mathrm{d} \vartheta}{\mathrm{d} y}\right|_{y=0} ; \quad q_{H}^{\prime \prime}=\left.\lambda_{\text {ref }} \frac{\mathrm{d} \vartheta}{\mathrm{d} y}\right|_{y=H} \\
\vartheta(0)=0
\end{gathered}
$$

It is worth remarking that the problem is non-linear due to the appearance of the $u / u_{m}$ source term in (18b). The boundary condition (18e) results from the definition of $\vartheta$. The flux boundary conditions (18d) and (18e) are not independent, as they are coupled through the global energy balance, resulting by integration of equation (18b) over the channel height. This constraint will be used to evaluate $u_{m}$, which enters the problem as an additional unknown. 


\subsection{Non-dimensional formulation}

The following non-dimensional quantities are introduced:

$$
X=\frac{x}{H} ; \quad Y=\frac{y}{H} ; \quad U=\frac{u}{U_{*}} ; \quad P=\frac{\widetilde{p}}{p_{*}} ; \quad \Theta=\frac{\vartheta}{T_{*}}
$$

where the velocity, pressure and temperature scales are defined as follows

$$
U_{*}=\frac{v_{g}^{2} H}{\nu_{\text {ref }}} ; \quad p_{*}=\rho_{\text {ref }} \beta_{\text {ref }} g \frac{\mathrm{d} T_{b}}{\mathrm{~d} x} H^{2} ; \quad T_{*}=\frac{\left|q_{0}^{\prime \prime}+q_{H}^{\prime \prime}\right|}{\lambda_{\text {ref }}} H
$$

Using equation (6), the non dimensional bulk temperature gradient in axial direction is

$$
\left|\frac{\mathrm{d} \Theta_{b}}{\mathrm{~d} X}\right|=\frac{1}{\mathrm{Ra}_{*} U_{m}}
$$

where the Rayleigh number $\mathrm{Ra}_{*}$ based on the temperature scale $T_{*}$ is defined as

$$
\mathrm{Ra}_{*} \equiv \frac{\beta_{\mathrm{ref}} g T_{*} H^{3}}{\nu_{\mathrm{ref}} \kappa_{\mathrm{ref}}}=\frac{U_{*} H}{\kappa_{\mathrm{ref}}}
$$

The governing equations in non-dimensional form result in:

$$
\begin{gathered}
\frac{\mathrm{d}^{2} U}{\mathrm{~d} Y^{2}}+\Theta \sin (\varphi)=\frac{\cos (\varphi)}{\operatorname{Ra}_{*} U_{m}}\left(Y-\frac{1}{2}\right) \\
\frac{\partial P}{\partial Y}=\left(\Theta \operatorname{Ra}_{*} U_{m}+X\right) \cos (\varphi) \\
\frac{\mathrm{d}^{2} \Theta}{\mathrm{d} Y^{2}}-\frac{U}{U_{m}}=0
\end{gathered}
$$

Equations (23a) - (23c) are complemented with the following boundary conditions

$$
\begin{gathered}
U(0)=0 ; \quad U(1)=0 \\
\left.\frac{\mathrm{d} \Theta}{\mathrm{d} Y}\right|_{Y=0}=-m ;\left.\quad \frac{\mathrm{d} \Theta}{\mathrm{d} Y}\right|_{Y=1}=1-m \\
\Theta(0)=0
\end{gathered}
$$

where $m$ is the ratio between the lower wall heat flux and the overall heat flux

$$
m \equiv \frac{q_{0}^{\prime \prime}}{q_{0}^{\prime \prime}+q_{H}^{\prime \prime}} ; \quad m \in(-\infty,+\infty)
$$


When the right hand side of equation (23a) is negligible with respect to the other terms, i.e. for

$$
\left|\cos (\varphi) /\left(\operatorname{Ra}_{*} U_{m}\right)\right| \ll\left|\mathrm{d}^{2} U / \mathrm{d} Y^{2}\right|
$$

and

$$
\left|\cos (\varphi) /\left(\operatorname{Ra}_{*} U_{m}\right)\right| \ll|\Theta \sin (\varphi)|
$$

equations (23a) and (23c) form a simplified system of ODEs for $U$ and $\Theta$ :

$$
\begin{gathered}
\frac{\mathrm{d}^{2} U}{\mathrm{~d} Y^{2}}+\Theta \sin (\varphi)=0 \\
\frac{\mathrm{d}^{2} \Theta}{\mathrm{d} Y^{2}}-\frac{U}{U_{m}}=0
\end{gathered}
$$

As already recognized in Piller and Stalio [15], problem (26) is satisfied by a set of self-similar solutions:

$$
\begin{gathered}
U_{\varphi}(Y)=U_{\frac{\pi}{2}}(Y) \sin (\varphi) \\
\Theta_{\varphi}(Y)=\Theta_{\frac{\pi}{2}}(Y)
\end{gathered}
$$

where subscript $\phi$ indicates quantities calculated for a channel inclined by $\varphi$ with respect to the horizontal, while subscript $\pi / 2$ indicates a vertical channel.

The assumptions leading to system (26) may be cast as ${ }^{1}$ :

$$
\frac{\operatorname{Ra}_{*} U_{m}^{2}}{|\cos (\varphi)|} \gg 1
$$

and

$$
\left|\Theta(1) \tan (\varphi) U_{m}\right| \mathrm{Ra}_{*} \gg 1
$$

Conditions (28) and (29) and thus equations (26) hold for nearly vertical channels, for large $\mathrm{Ra}_{*}$ or for large $U_{m}$, but still within the stationary, laminar flow regime.

$$
{ }^{1} \mathrm{~d}^{2} U / \mathrm{d} Y^{2} \sim U_{m}
$$




\subsection{Uncoupled equations for $U$ and $\Theta$}

Problem (23) and (24) can be reduced to the following set of uncoupled fourth-order ordinary differential equations

$$
U^{\prime \prime \prime \prime}+\frac{U}{U_{m}} \sin (\varphi)=0
$$

with boundary conditions

$$
\begin{aligned}
& U(0)=U(1)=0 ; \quad U^{\prime \prime}(0)=-\frac{1}{2} \frac{\cos (\varphi)}{\operatorname{Ra}_{*} U_{m}} ; \\
& U^{\prime \prime \prime}(0)=m \sin (\varphi)+\frac{\cos (\varphi)}{\operatorname{Ra}_{*} U_{m}} ; \\
& U^{\prime \prime \prime}(1)=(m-1) \sin (\varphi)+\frac{\cos (\varphi)}{\operatorname{Ra}_{*} U_{m}}
\end{aligned}
$$

and

$$
\Theta^{\prime \prime \prime \prime}+\frac{\Theta}{U_{m}} \sin (\varphi)=\frac{\cos (\varphi)}{\operatorname{Ra}_{*} U_{m}^{2}}\left(Y-\frac{1}{2}\right)
$$

with boundary conditions

$$
\Theta(0)=0 ; \quad \Theta^{\prime}(0)=-m ; \quad \Theta^{\prime}(1)=1-m ; \quad \Theta^{\prime \prime}(0)=\Theta^{\prime \prime}(1)=0
$$

A particular solution for the linear, ordinary differential equation (31a) is

$$
\Theta_{p}(Y)=\frac{1}{\operatorname{Ra}_{*} U_{m} \tan \varphi}\left(Y-\frac{1}{2}\right)
$$

which can be rewritten as

$$
\Theta_{p}(Y)=\frac{\cos (\varphi)}{\operatorname{Ra}_{*}(\sin (\varphi))^{2}} \frac{\sin (\varphi)}{U_{m}}\left(Y-\frac{1}{2}\right)
$$

By inspection of equation (31a), (31b) and (33), it can be deduced that the solutions for $\Theta(Y)$ and for $U_{m} / \sin (\varphi)$ depend on the only free parameter

$$
A \equiv \frac{1}{\mathrm{Ra}_{*}} \frac{\cos (\varphi)}{(\sin (\varphi))^{2}}
$$

which includes information about heating conditions and channel inclination. The following discussion is for $A \neq 0$. 
Assuming $U_{m}>0$ the solution to problem (31) is given by

$$
\begin{aligned}
\Theta(Y) & =\exp (\alpha Y)[a \sin (\alpha Y)+b \cos (\alpha Y)] \\
& +\exp (-\alpha Y)[c \sin (\alpha Y)+d \cos (\alpha Y)] \\
& +4 \alpha^{4} A\left(Y-\frac{1}{2}\right)
\end{aligned}
$$

where

$$
\alpha \equiv \sqrt[4]{\frac{\sin (\varphi)}{4 U_{m}}}
$$

Assuming $U_{m}<0$ the general solution to problem (31) is

$$
\begin{aligned}
\Theta(Y) & =a \exp (-\sigma y)+b \exp (\sigma y)+c \sin (\sigma y)+d \cos (\sigma y)+ \\
& +\sigma^{4} A\left(Y-\frac{1}{2}\right)
\end{aligned}
$$

where

$$
\sigma \equiv \sqrt[4]{\frac{\sin (\varphi)}{\left|U_{m}\right|}}
$$

\subsection{Equation for the pressure field}

Integration of equation (23b) between 0 and $Y$ gives

$$
P(X, Y)=\left[U_{m} \operatorname{Ra}_{*} \int_{0}^{Y} \Theta(\widehat{Y}) \mathrm{d} \widehat{Y}+X Y\right] \cos (\varphi)+g(X)
$$

where $g(X)$ denotes an unknown function of $X$. The $Y$-averaged value of $P$ is

$$
\bar{P}(X)=\left[U_{m} \operatorname{Ra}_{*} \int_{0}^{1} \int_{0}^{Y} \Theta(\widehat{Y}) \mathrm{d} \widehat{Y} \mathrm{~d} Y+\frac{X}{2}\right] \cos (\varphi)+g(X)
$$

It follows from equation (15) that

$$
\frac{\partial \bar{P}}{\partial X}=g^{\prime}(X)+\frac{1}{2} \cos (\varphi)=0 \Longrightarrow g(X)=-\frac{X}{2} \cos (\varphi)+P(0,0)
$$

yielding

$$
P(X, Y)=P(0,0)+\left[U_{m} \operatorname{Ra}_{*} \int_{0}^{Y} \Theta(\widehat{Y}) \mathrm{d} \widehat{Y}+X\left(Y-\frac{1}{2}\right)\right] \cos (\varphi)
$$




\subsection{Integral inequalities}

The non dimensional momentum flux $E_{U}$ and the enthalpy flux $\Phi_{\Theta}$ are defined as

$$
\begin{gathered}
E_{U} \equiv \int_{0}^{1}(U(Y))^{2} \mathrm{~d} Y \\
\Phi_{\Theta} \equiv \int_{0}^{1} U(Y) \Theta(Y) \mathrm{d} Y
\end{gathered}
$$

In analogy to equation (43), the mean, squared temperature $E_{\Theta}$ is defined as

$$
E_{\Theta} \equiv \int_{0}^{1}(\Theta(Y))^{2} \mathrm{~d} Y
$$

Taking the inner product (in $\mathbb{L}^{2}([0,1])$ ) of equation (23a) for the vertical channel with $U$ yields, upon integration by parts and using the boundary conditions:

$$
\Phi_{\Theta}=\int_{0}^{1}\left(U^{\prime}\right)^{2} \mathrm{~d} Y
$$

which implies also $\Phi_{\Theta} \geq 0$.

Using the Poincaré's inequality [16] yields

$$
E_{U} \leq \int_{0}^{1}\left(U^{\prime}\right)^{2} \mathrm{~d} Y=\Phi_{\Theta}
$$

The inequality $E_{U} \leq \Phi_{\Theta}$ is prone to a physical interpretation: in the free-convection flow of the present investigation, momentum flux is due to the axial component of the buoyancy force, whose establishment requires an axial enthalpy flux. The enthalpy flux thus provides an upper bound for the momentum flux.

\section{Results and discussion}

Equations (35) and (37) supplemented by boundary conditions lead to the solutions to problem (31). The heat transfer configuration selected for the present study is non-symmetric, where the $Y=1$ wall is heated while the $Y=0$ wall is adiabatic and therefore the heating ratio is $m=0$. 
Details on the evaluation of constants in equations (35) and (37) are provided in Appendix A and Appendix B.

As in some ranges of the parameter $A$ infinite solutions are found, the discussion is sometimes limited to the first-order solution, meant as the solution corresponding to the largest flow rate $\left|U_{m}\right|$.

\subsection{First-order solution for positive mean velocity and $m=0$}

Scaled temperature and velocity profiles corresponding to a set of first roots $\alpha_{0}$ obtained for selected values of the parameter $A$ are shown in figures 2 and 3 . For $0<A \lesssim 0.03$, the velocity field does not show any flow reversal; for $A \gtrsim 0.03$ flow reversal is seen to occur. Two limiting conditions are represented in figure 2 by the $A=10^{-5}$ and the $A=10^{7}$ cases, these correspond to the balance between viscous and buoyancy forces in axial direction and to the balance between pressure and viscous forces, respectively. As explained in further detail in section 3.3, the tendency towards an antisymmetric velocity profile is determined by the pressure term of equation (23a) being dominant with respect to buoyancy.

The temperature profile, which is convex for small values of the parameter $A$, displays an inflection point close to the upper wall as soon as the flow is reversed in the same region. The inflection point migrates form the upper wall towards the channel centerline with increasing $A$, see figure 3 .

Derivatives in $x$ and $y$ direction of the pressure field divided by $\cos (\varphi)$ are displayed in figure 4 for both $A>0$ (top wall heated) and $A<0$ (bottom wall heated).

Multiple solutions for almost vertical channels. As long as the parameter $A$ is positive and not very small, only one solution to problem (30) and (31) exists. Instead, for $A \lesssim 3 \times 10^{-7}$ multiple solutions are found; consider that $A \mathrm{Ra}_{*}<10^{-7}$ only for $\varphi=(1-\epsilon) \pi / 2$ where $0<\epsilon \lesssim 6 \times 10^{-7}$. The three solutions to problem (31) for $A=10^{-8}$ and $U_{m}>0$ are shown in figure 5 , where it is noticed that increasing the order of solution, the number of flow reversals is increased as well. In summary and for the $A>0$ case, only one solution to problem (31) exists unless $A$ is very small, i.e. unless the channel is almost vertical or for very large values of $\operatorname{Ra}_{*}$. For $\varphi$ between $(1-\epsilon) \pi / 2<\varphi<\pi / 2$ the number of solutions increases by two at a time as $\epsilon \rightarrow 0$.

The multiplicity of solutions is a noteworthy feature of the considered natural convection flow configuration, described by non-linear, steady equa- 


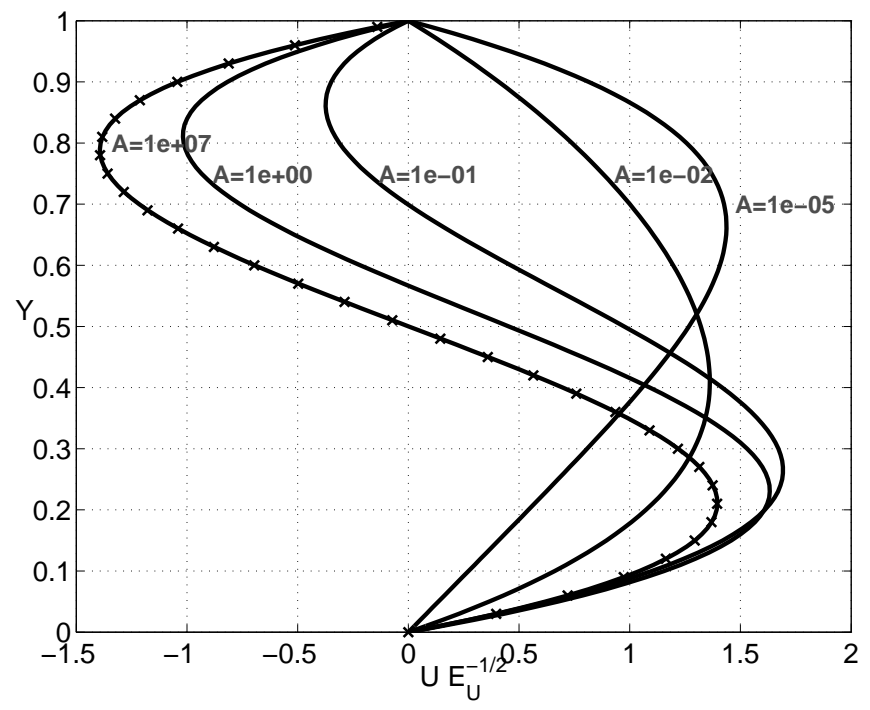

Figure 2: Scaled velocity profiles for different $A$ values between $A=10^{-5}$ and $A=10^{7}$; x-symbols indicate the limiting solution, equation (51). The momentum flux $E_{U}$ used for scaling is defined in equation (43).

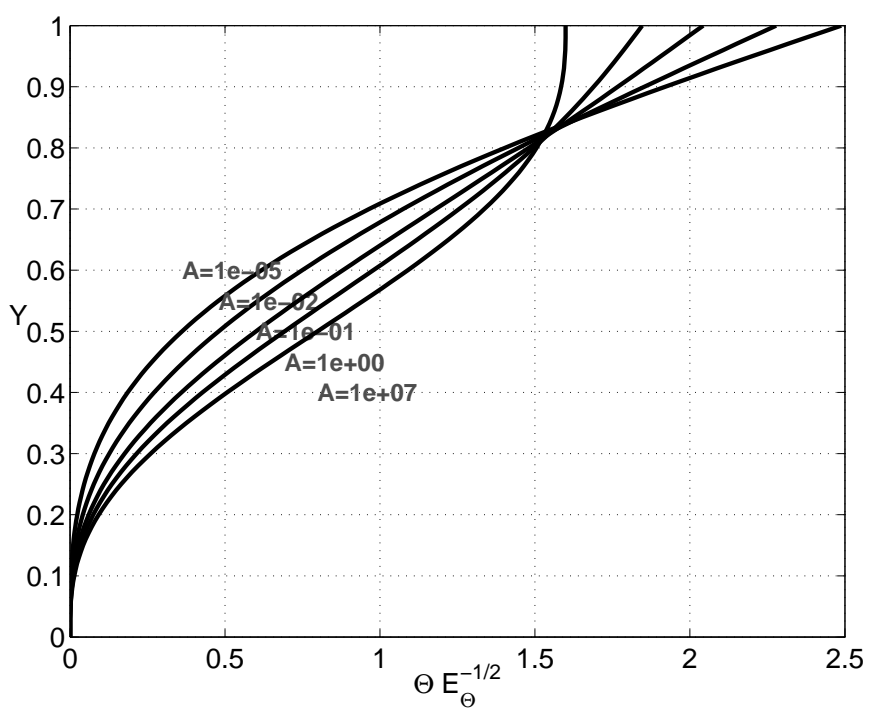

Figure 3: Scaled temperature profiles for different $A$ values between $A=10^{-5}$ and $A=$ $10^{7}$. The averaged square temperature $E_{\Theta}$ used for scaling is defined in equation (45). 


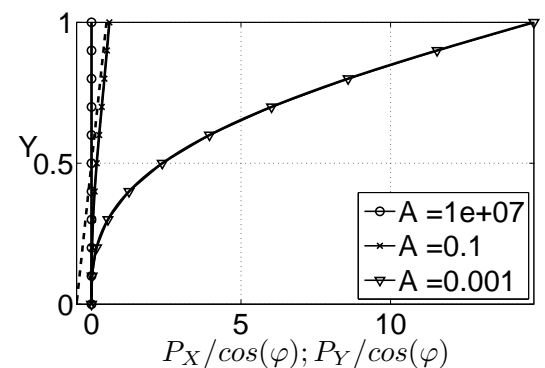

(a) $\varphi=\frac{1}{12} \pi$

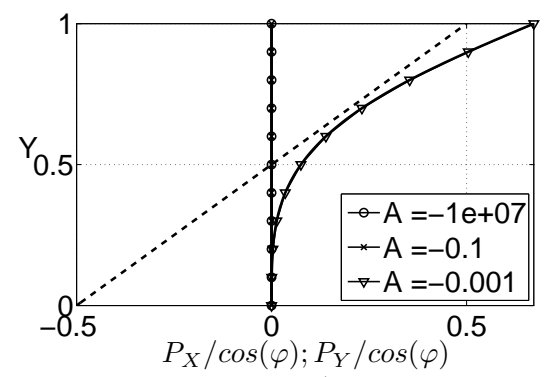

(c) $\varphi=\frac{7}{12} \pi$

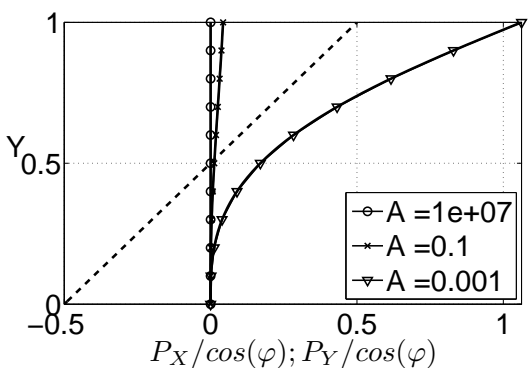

(b) $\varphi=\frac{5}{12} \pi$

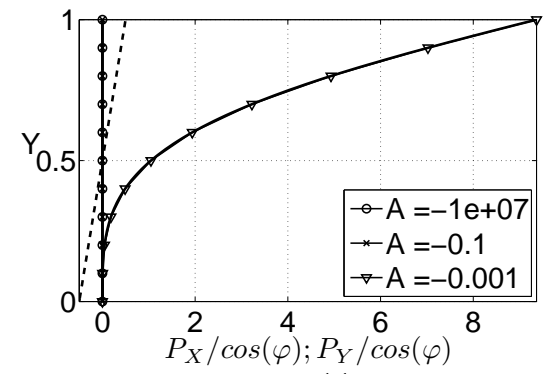

(d) $\varphi=\frac{11}{12} \pi$

Figure 4: Pressure field derivatives for different inclination angles. Dashed lines: $x$ derivative of $P / \cos (\varphi)$. Lines with symbols: $y$-derivative of $P / \cos (\varphi)$ for three different different $A$ values.

tions. When governing equations are linear, they typically yield one single base-state solution, as in the work by Bergholz [4]. A multiplicity of coexisting base-state solutions are instead found in Ref. [5], where the flow is governed by non-linear conservation equations. In the present work, multiple solutions are determined solving analytically the steady conservation equations, which would be interpreted as coexisting base-state solutions (either stable or unstable) in the numerical investigation of the corresponding time-dependent equations [5].

Solutions for $A<0$ and $U_{m}>0$. Solutions can be found for negative $A$ provided $|A|$ is small enough, $|A| \lesssim 1.1 \times 10^{-3}$. Negative values for $A$ correspond to inclination angles $\pi / 2<\varphi<\pi$ and thus to the case where the heated plate is at the bottom (unstably-stratified flow). Velocity and temperature profiles of the first order solutions are displayed in figure 6 . Flow inversion is observed from $|A| \lesssim 3 \times 10^{-4}$. As might be inferred from the figure, transi- 


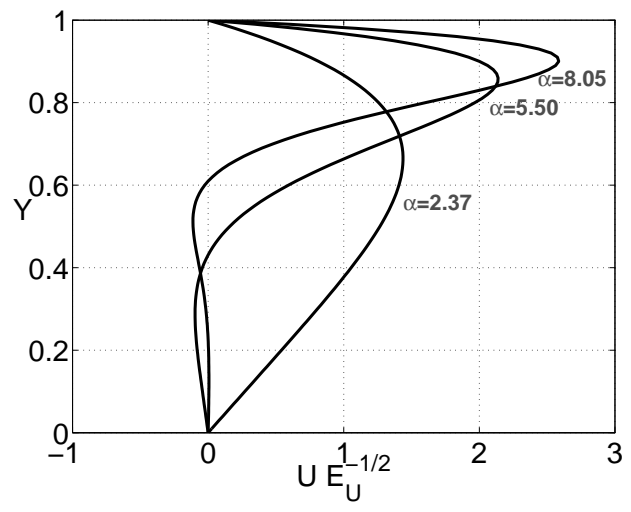

(a) Velocity profiles

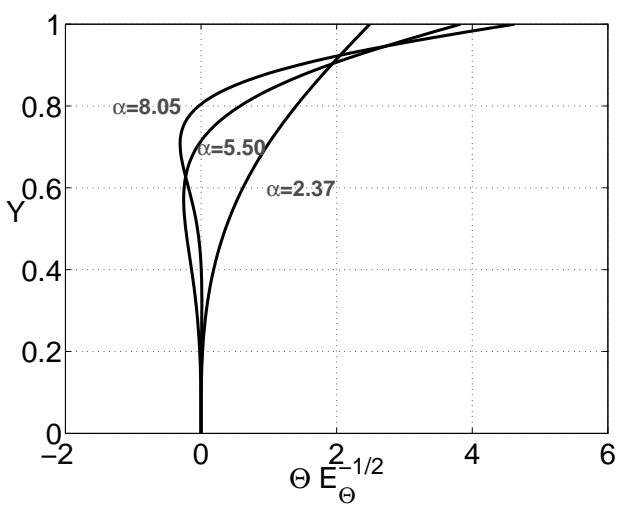

(b) Temperature profiles

Figure 5: Multiple solutions for velocity and temperature, $A=10^{-8}$ and the three roots of equation (67), $\alpha_{0}=2.3650196368785, \alpha_{1}=5.5033828009739, \alpha_{2}=8.045898882801911$.

tion between concave velocity profile and velocity profile displaying one flow reversal is smooth; this feature is confirmed by the heat transfer maps in section 3.4.2.

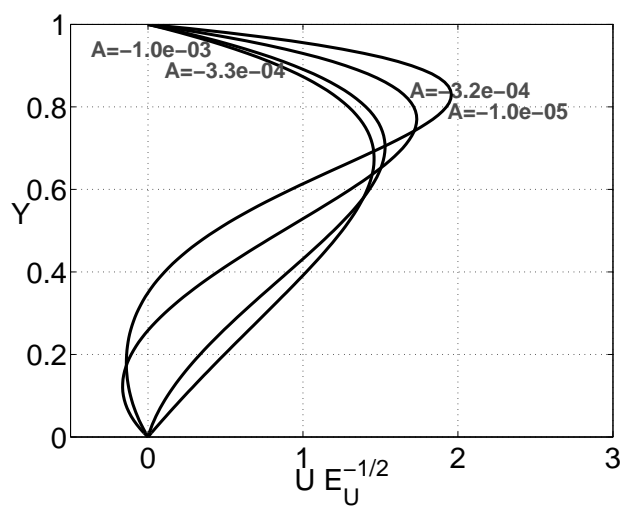

(a) Velocity profiles

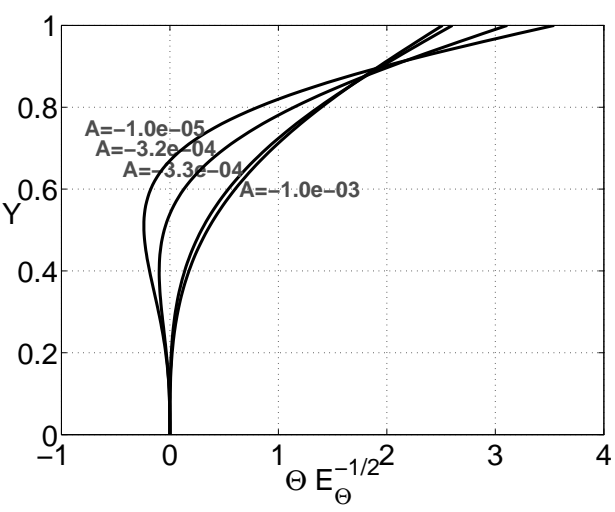

(b) Temperature profiles

Figure 6: Velocity and temperature profiles of the first order solutions for negative $A$ and positive $U_{m}$.

\subsection{First-order solution for negative mean velocity and $m=0$}

Velocity and temperature profiles for the first-order $\sigma_{1}$ root for $A>0$ identify two critical $A$ values across which the flow pattern changes discon- 


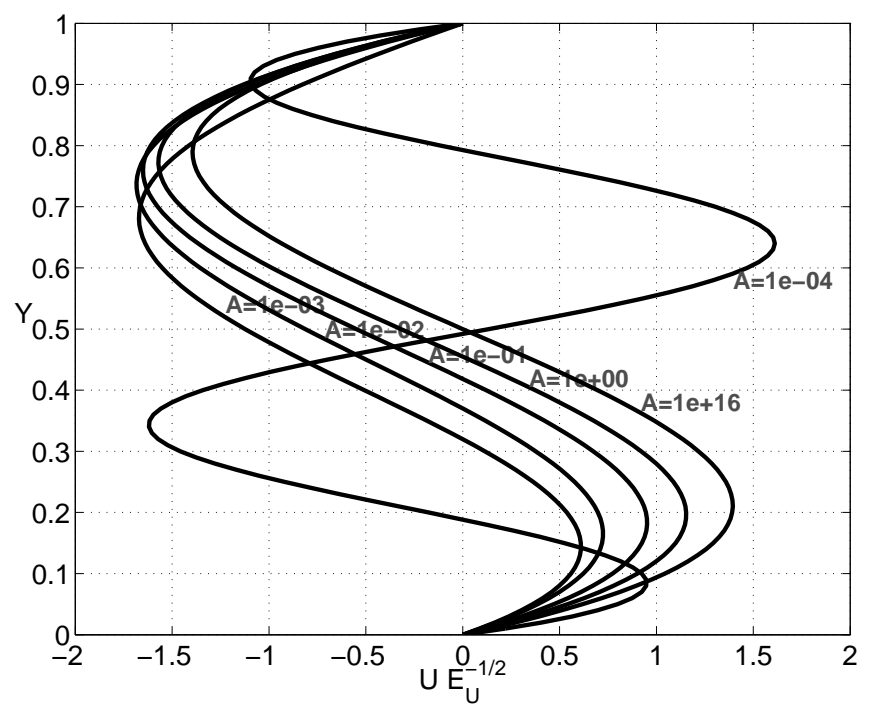

Figure 7: Scaled velocity profiles for $\sigma_{1}(A)$ solutions with different $A$ values between $A=10^{-3}$ and $A=10^{16}$. The momentum flux $E_{U}$ used for scaling is defined in equation (43).

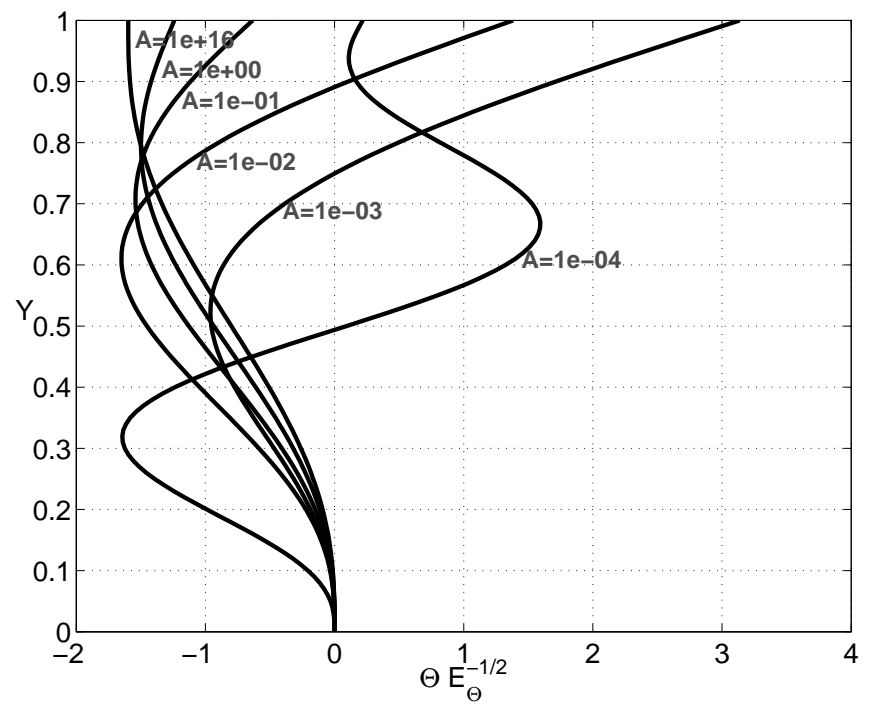

Figure 8: Scaled temperature profiles for $\sigma_{1}(A)$ solutions with different $A$ values between $A=10^{-3}$ and $A=10^{16}$ The averaged square temperature $E_{\Theta}$ used for scaling is defined in equation (45). 


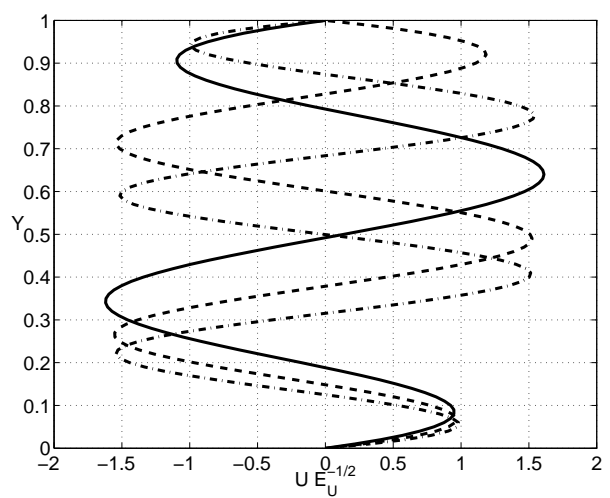

(a) Velocity profiles, $A=10^{-4}$

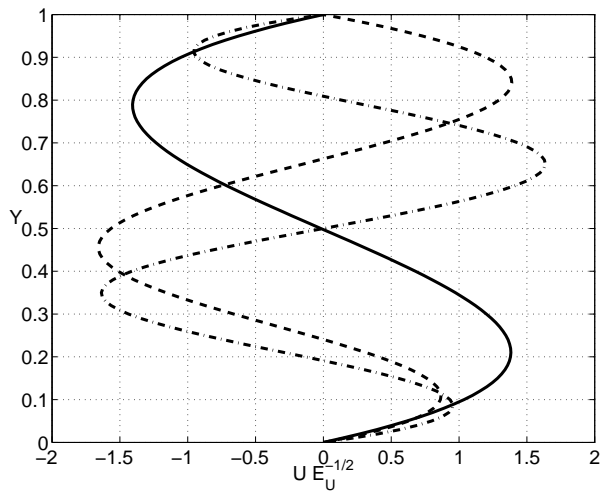

(c) Velocity profiles, $A=10^{4}$

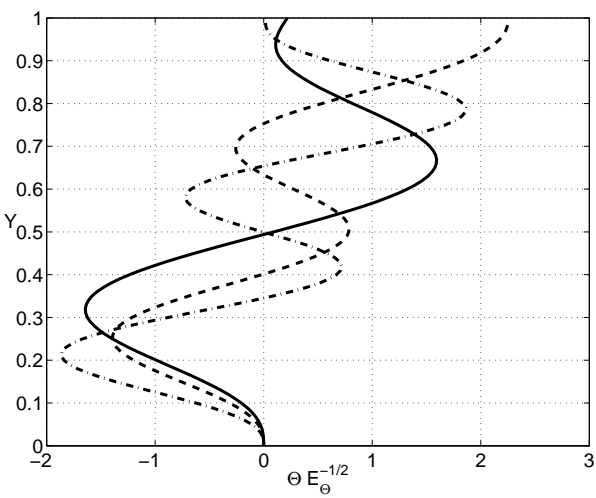

(b) Temperature profiles, $A=10^{-4}$

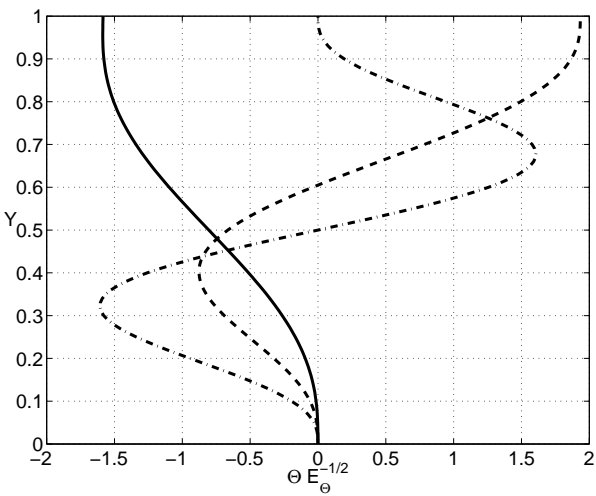

(d) Temperature profiles, $A=10^{4}$

Figure 9: Multiple solutions for $A=10^{-4}$ and $A=10^{4}$. Solid lines: solution corresponding to $\sigma_{1}$; Dashed lines: solution corresponding to $\sigma_{2}$; Dash-dot lines: solution corresponding to $\sigma_{3}$.

tinuously, these correspond to $\log _{10}\left(A_{c, 1}\right) \approx-5.25$ and $\log _{10}\left(A_{c, 2}\right) \approx-3.748$. The number of flow reversals decreases from 5 to 3 when $A$ is increased across $A_{c, 1}$, it instead decreases from 3 to 1 for increasing $A$ across $A_{c, 2}$; the number of inflection points in the $\Theta$ profile changes accordingly. For very large values of $A$ the flow becomes anti-symmetric and the temperature field is negative throughout the channel. Scaled profiles for the first-order root are displayed in figures 7 and 8 , where $A \geq 10^{-4}$ for clarity. Higher-order solutions are shown in figure 9 .

Also in the case of negative $U_{m}$, solutions can be found for $A<0$, where the heated plate is at the bottom. Velocity and temperature profiles of 
the first-order solutions are displayed in figure 10. For large $|A|$ values, the velocity field is characterized by two flow reversals. Two critical points are identified for smaller $|A|$, corresponding to $\log _{10}\left(-A_{c, 3}\right) \approx-4.3$ and $\log _{10}\left(-A_{c, 4}\right) \approx-5.55$. The number of flow reversals and the number of inflection points in the $\Theta$ profiles decrease from 6 to 4 when $|A|$ is decreased across $\left|A_{c, 3}\right|$ and from 4 to 2 when $|A|$ is decreased across $\left|A_{c, 4}\right|$. For clarity figure 10 displays only $|A| \geq 10^{-5}$ profiles.

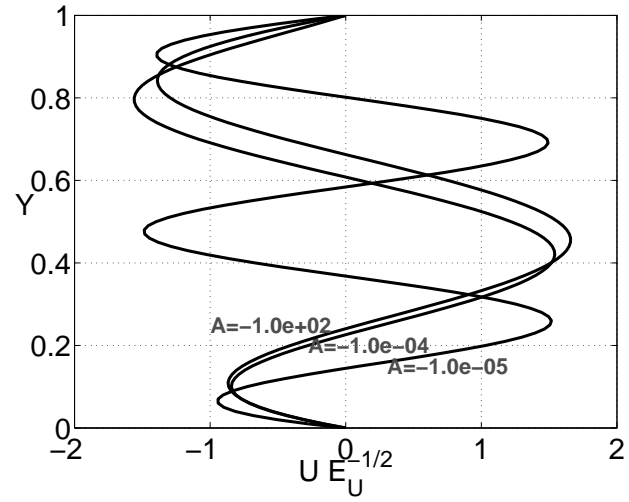

(a) Velocity profiles

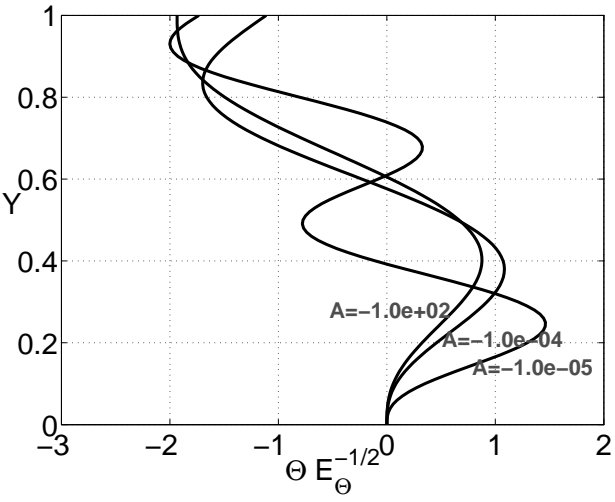

(b) Temperature profiles

Figure 10: Velocity and temperature profiles of the first order solutions for negative $A$ and negative $U_{m}$.

\subsection{Analysis of the mechanism leading to flow inversion}

In this section, the mechanism leading to flow inversion is discussed. The analysis is carried out for the $m=0$ heat transfer configuration.

When $U_{m}>0$, equation (23a) may be recast as

$$
U^{\prime \prime}+\left[\Theta-4 A \alpha^{4}\left(Y-\frac{1}{2}\right)\right] \sin (\varphi)=0
$$

where three terms are identified: the viscous force term $F_{v}=U^{\prime \prime}$, the $x$ component of the buoyancy force induced by temperature differences in wall normal direction $F_{\Theta}=\Theta \sin (\varphi)$ and

$$
F_{p}=-4 A \alpha^{4}\left(Y-\frac{1}{2}\right) \sin (\varphi)
$$


which can be interpreted as the force due to the pressure gradient $\partial \widetilde{p} / \partial x$, whose $y$-variation is to be ascribed to the axial variation of the wall-normal buoyancy force in equation $(9 \mathrm{~b})$. It is worth remarking that $F_{p}$ is antisymmetric. Figure 2 shows that for very large values of $A$ this behavior is inherited by the velocity profile.

The basic assumption of the following analysis is that, for large $A$

$$
\left|4 A \alpha^{4}\left(Y-\frac{1}{2}\right)\right| \gg|\Theta|
$$

except possibly for a small region around the channel centerline. Notice that as $\Theta$ depends on $A$, this assumption will have to be confirmed. Once more, only the lower order solutions (corresponding to $\alpha_{0}$ for $U_{m}>0$ ) are considered.

Equation (48) for very large values of $A$ and assuming that constraint (50) holds leads to the following solution

$$
U^{(p)}(Y)=\frac{C}{6}\left(Y-\frac{1}{2}\right)\left[\left(Y-\frac{1}{2}\right)^{2}-\frac{1}{4}\right]
$$

where

$$
C \equiv 4 A \alpha^{4} \sin (\varphi)
$$

The momentum flux defined in (43) results in

$$
E_{U}=\frac{C}{30240}
$$

and the normalized velocity profile is

$$
U^{(p)} E_{U}^{-\frac{1}{2}}=\sqrt{840}\left[\left(Y-\frac{1}{2}\right)^{2}-\frac{1}{4}\right]\left(Y-\frac{1}{2}\right)
$$

The $U^{(p)} E_{U}^{-\frac{1}{2}}$ profile is represented by x-symbols on figure 2. Visual comparison provides confirmation that equation (54) is the limit of $U E_{U}^{-\frac{1}{2}}$ for $A \rightarrow+\infty$. A similar analysis conducted for the $U_{m}<0$ case leads to a velocity profile $U^{(n)}$ for large $A$, which is the opposite of $U^{(p)}$

$$
U^{(n)}=-U^{(p)}
$$

Althought not reported for brevity, it has been verified that for $A \gtrsim 0.02$ the energy of $F_{p}$, defined as $\int_{0}^{1} F_{p}^{2} \mathrm{~d} Y$, becomes larger than the averaged square temperature $E_{\Theta}$, for both $U_{m}>0$ and $U_{m}<0$. 


\subsection{Overview of flow configurations and heat transfer rates}

\subsubsection{Overview of flow configurations}

Sections 3.1 and 3.2 identify different flow and temperature field features corresponding to adjacent ranges of values for the $A$ parameter. In some cases abrupt changes occur across critical values of $A$. In order to summarize these critical points together with the main features of solutions found, tables 1 and 2 collect flow patterns and temperature profile characteristics for $U_{m}>0$ or $U_{m}<0$, with either $A>0$ or $A<0$. The number of flow inversions $n_{i}$ is provided for each case because in the limit as $\operatorname{Pr} \rightarrow 0$, an inflectional velocity profile in a plane parallel flow is a necessary condition for instability [13].

\begin{tabular}{|l|l|l|l|l|}
\hline \multicolumn{5}{|c|}{$U_{m}>0 ; A>0$} \\
\hline$A$ & $n_{s}$ & $n_{i}$ for $\alpha_{0}$ & $\Theta(1)$ for $\alpha_{0}$ & $\Theta(Y)$ for $\alpha_{0}$ \\
\hline $0<A \lesssim 3 \times 10^{-7}$ & $3,5,7 \ldots$ & 0 & $>0$ & $>0$ \\
$3 \times 10^{-7}<A \lesssim 3 \times 10^{-2}$ & 1 & 0 & $>0$ & $>0$ \\
$3 \times 10^{-2} \lesssim A$ & 1 & 1 & $>0$ & $>0$ \\
\hline \multicolumn{5}{|c|}{$U_{m}>0 ; A<0$} \\
\hline$|A|$ & $n_{s}$ & $n_{i}$ for $\alpha_{0}$ & $\Theta(1)$ for $\alpha_{0}$ & $\Theta(Y)$ for $\alpha_{0}$ \\
\hline $0<|A| \lesssim 3.3 \times 10^{-4}$ & $2,4,6 \ldots$ & 1 & $>0$ & $\lessgtr 0$ \\
$3.3 \times 10^{-4} \lesssim|A| \lesssim 1.1 \times 10^{-3}$ & 2 & 0 & $>0$ & $>0$ \\
$1.1 \times 10^{-3} \lesssim|A|$ & 0 & - & - & - \\
\hline
\end{tabular}

Table 1: Summary of flow and temperature field features for $U_{m}>0$. In the table $n_{s}$ indicates the number of solutions, $n_{i}$ the number of flow reversals.

\begin{tabular}{|c|c|c|c|c|}
\hline \multicolumn{5}{|c|}{$U_{m}<0 ; A>0$} \\
\hline$A$ & $n_{s}$ & $n_{i}$ for $\sigma_{1}$ & $\Theta(1)$ for $\sigma_{1}$ & $\Theta(Y)$ for $\sigma_{1}$ \\
\hline $0 \lesssim A \lesssim 5.6 \times 10^{-6}$ & $\infty$ & 5 & $>0$ & $\$ 0$ \\
\hline $5 . \widetilde{6} \times 10^{-6} \lesssim A \lesssim 1.8 \times 10^{-4}$ & $\infty$ & 3 & $>0$ & $\lessgtr 0$ \\
\hline $1.8 \times 10^{-4} \underset{\approx}{\approx} A$ & $\infty$ & 1 & $\lessgtr 0$ & $\lessgtr 0$ \\
\hline \multicolumn{5}{|c|}{$U_{m}<0 ; A<0$} \\
\hline$|A|$ & $n_{s}$ & $n_{i}$ for $\sigma_{1}$ & $\Theta(1)$ for $\sigma_{1}$ & $\Theta(Y)$ for $\sigma_{1}$ \\
\hline $0 \lesssim|A| \lesssim 2.8 \times 10^{-6}$ & $\infty$ & 6 & $<0$ & $\lessgtr 0$ \\
\hline $2.8 \times 10^{-6} \lesssim|A| \lesssim 5.0 \times 10^{-5}$ & $\infty$ & 4 & $<0$ & $\lessgtr 0$ \\
\hline 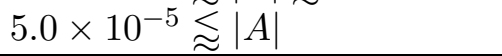 & $\infty$ & 2 & $<0$ & $\lessgtr 0$ \\
\hline
\end{tabular}

Table 2: Summary of flow and temperature field features for $U_{m}<0$. In the table $n_{s}$ indicates the number of solutions, $n_{i}$ the number of flow reversals. 


\subsubsection{Maps of heat transfer rates}

In the fully developed conditions of the present study, the Nusselt number is independent of $x$. The ratio between the actual heat flux $q_{H}^{\prime \prime}$ released to the flow and the pure-conduction heat flux $\lambda_{\text {ref }}\left(T(H)-T_{b}\right) / H$ provides a suitable indication of the heat transfer efficiency of the considered natural convection flow. Thus, the following definition of the Nusselt number is given

$$
\left.\mathrm{Nu} \equiv \frac{1}{\vartheta(H)-\vartheta_{b}} \frac{\partial \vartheta}{\partial y}\right|_{H}=\frac{1}{\Theta(1)-\Theta_{b}}
$$

Since the bulk temperature can be expressed as a function of the temperature profile

$$
\Theta_{b}=\frac{1}{U_{m}} \int_{0}^{1} U(Y) \Theta(Y) \mathrm{d} Y=\int_{0}^{1} \Theta^{\prime \prime}(Y) \Theta(Y) \mathrm{d} Y
$$

which in turns is a function of the parameter $A$ only, the following functional dependency holds

$$
\mathrm{Nu}=\mathrm{Nu}(A)
$$

this dependency is described in the following text, with reference to figures 11 and 12 .

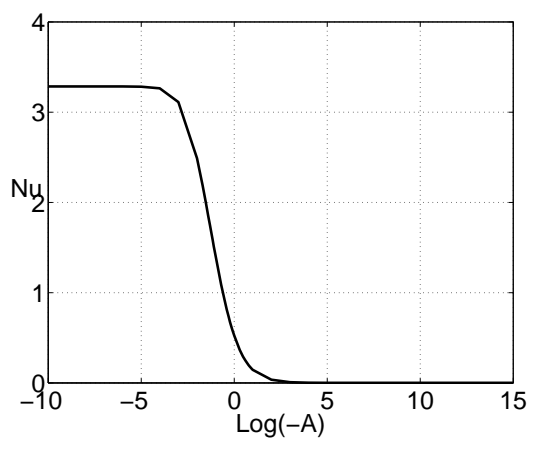

(a) $\mathrm{Nu}, A>0$

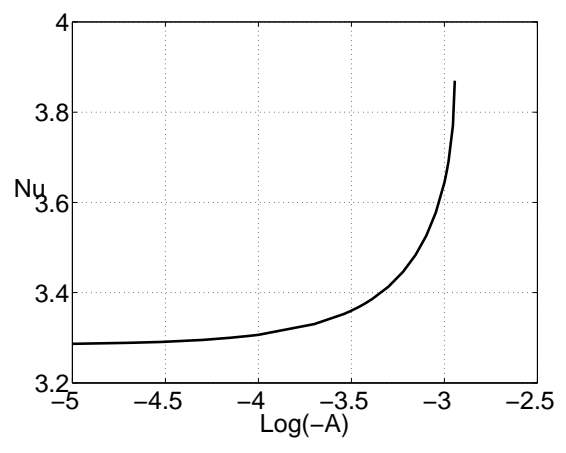

(b) $\mathrm{Nu}, A<0$

Figure 11: Nusselt number as functions of the parameter $A, U_{m}>0$.

$\boldsymbol{U}_{m}>0, \boldsymbol{A}>0$. The Nusselt number behavior for $U_{m}>0, A>0$ is depicted in figure 11(a). For small $A$ values, the Nusselt number is nearly constant and 


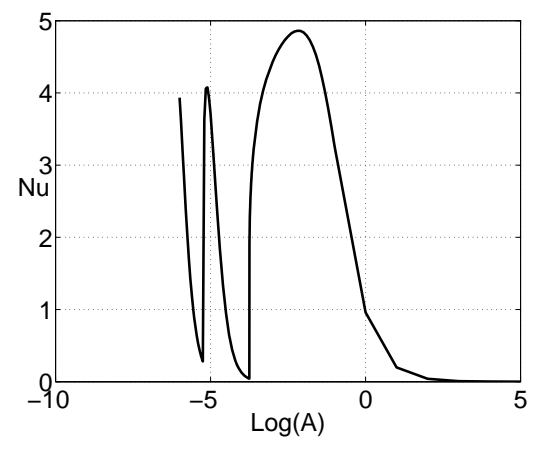

(a) $\mathrm{Nu}, A>0$

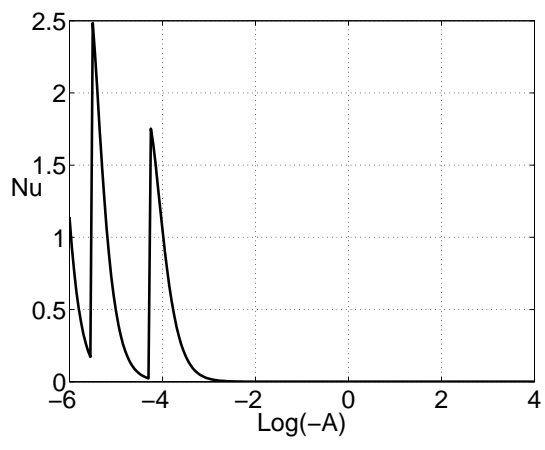

(b) $\mathrm{Nu}, A<0$

Figure 12: Nusselt number as functions of the parameter $A, U_{m}<0$.

$\mathrm{Nu} \approx 3.285$ for $A \rightarrow 0$. The appearance of the flow reversal shown in figure 2 about $A \approx 0.03$ is accompanied by a Nusselt number decrease. For fixed $\mathrm{Ra}_{*}$ this can be explained by weak advection effects for almost horizontal channels, due in turn to a small component of the buoyancy force along the streamwise direction.

$\boldsymbol{U}_{m}>0, \boldsymbol{A}<0$. Solutions exist in this case for $|A| \lesssim 1.1 \times 10^{-3}$. The Nusselt number increases continuously and monotonically all over the range of $A$ where solutions exist. The function $\mathrm{Nu}(A)$ is smooth also across the $|A| \approx 3 \times 10^{-4}$ value, separating the concave velocity profile range from the flow configurations displaying one flow reversal. For maximum $|A|$, the Nusselt number reaches $\mathrm{Nu} \approx 3.87$.

$\boldsymbol{U}_{m}<0, \boldsymbol{A}>0$. This case has infinitely many solutions; only the first order solution, corresponding to the maximum mean velocity is discussed here. The $\mathrm{Nu}(A)$ profile in figure $12(\mathrm{a})$ is characterized by critical $A$ values across which the solution changes discontinuously. These correspond to the critical points discussed in section 3.2 for the velocity and temperature profiles. For $\log _{10}(A) \approx-2.2, \mathrm{Nu}$ attains its maximum value, $\mathrm{Nu} \approx 4.86$. The flow configuration corresponding to maximum $\mathrm{Nu}$ is characterized by one flow reversal and one inflection point in the $\Theta$ profile.

$\boldsymbol{U}_{m}<0, \boldsymbol{A}<0$. Also in this case, of the infinite many solutions found, only the one corresponding to the maximum mean velocity is discussed. Two critical points are identified in the $\mathrm{Nu}(A)$ profile of figure $12(\mathrm{~b})$. The critical 
points correspond to those observed in the flow and temperature fields and discussed in section 3.2.

\subsection{Fully developed solutions as the result of flow and thermal development}

This section reports on several two dimensional numerical simulations of the hydraulically- and thermally-developing natural convection flow in long, inclined channels for a heating ratio $m=0$. The idea is that the most physical analytical solution between the multiple solutions found should correspond to the one obtained trough numerical simulation as a result of flow and thermal development.

Only the $U_{m}>0$ case is considered in this section as it was shown [12] that natural convection flows between inclined parallel plates under adverse temperature gradient (as in the case of $U_{m}<0$ ) are always unstable.

The aspect ratio of the two-dimensional channel employed for the simulations is $L / H=80$, so that fully developed conditions are reached well before the outlet region.

A uniform pressure is enforced at the inlet section, while the analytical pressure distribution is set at the outlet. The flow enters the heated section with uniform temperature $T_{0}=0$, which is used as reference temperature for the simulations. At the outlet, zero thermal axial diffusion is assumed.

While the following dimensionless groups are kept fixed for all simulations

$$
\mathrm{Ra}_{*}=0.1 ; \quad \operatorname{Pr}=1
$$

the inclination angle $\varphi$ is varied, in order to span a convenient range of the governing parameter $A$, as reported in table 3 .

The numerical simulations are carried out with the finite-element commercial software COMSOL Multiphysics ${ }^{\circledR}$. Unequal-order shape elements are used, quadratic for the velocity and linear for the pressure. Based on a grid-independence study, it is recognized that a good compromise between accuracy and computational efficiency is attained by using by a Cartesian mesh composed of equal elements of size $0.1 \times 0.02$ in the streamwise and wall-normal directions, respectively.

Numerical results for the global thermal energy, $E_{\Theta}$ and the mean velocity $U_{m}$ are given in table 3 , together with the percent error between the numerical and the analytical results corresponding to the lowest-order solution, $\alpha_{0}$. The

far-field numerical results agree with the analytical results corresponding to $\alpha_{0}$. 


\begin{tabular}{llll}
\hline$\varphi(\mathrm{deg})$ & $A$ & $U_{m} / \sin (\varphi)$ & $E_{\Theta}$ \\
\hline 90 & 0 & $0.007901(-1.1 \%)$ & $0.02973(-2.70 \%)$ \\
89.9999999 & $10^{-8}$ & $0.007889(-1.3 \%)$ & $0.02973(-0.55 \%)$ \\
89.9999000 & $1.5708 \times 10^{-5}$ & $0.007889(-1.5 \%)$ & $0.02978(-0.61 \%)$ \\
89.9990000 & $1.5708 \times 10^{-4}$ & $0.007891(-3.1 \%)$ & $0.03030(-1.1 \%)$ \\
89.9900000 & $1.5708 \times 10^{-3}$ & $0.009149(-0.16 \%)$ & $0.03627(0.67 \%)$ \\
89.9000000 & $1.5708 \times 10^{-2}$ & $0.012907(-3.4 \%)$ & $0.06329(0.32 \%)$ \\
\hline
\end{tabular}

Table 3: Numerical results for two-dimensional developing flow and heat transfer. Numbers reported within brackets report the percent error with respect to the analytical result.

The results of the test performed can be summarized as follows

- In all simulated cases the fully developed profiles extracted approximate very closely analytical results, see table 3.

- For the case $A=10^{-8}$ where the three solutions of figure 9 are found analytically, the numerical solution invariably converges towards the lowest order solution $\alpha_{0}$. Notice that this occurs also when initial conditions provided to the numerical code correspond to the $\alpha_{1}$ or $\alpha_{2}$ velocity and temperature fields.

- The same is verified also for the vertical channel $A=0$, whose infinite many analytical solutions are not provided here.

Numerical results support the hypothesis that between the multiple analytical solutions obtained for very small values of the parameter $A$, the stable solution is the lowest order solution, which is characterized by the smallest positive root $\alpha=\alpha_{0}$ and by the minimum number of flow reversals.

\section{Concluding Remarks}

The mathematical model representing the fully-developed, laminar free convection between inclined parallel plates is introduced and solved analytically. One wall is uniformly heated while the other is assumed adiabatic. Both cases of stable and unstable stratification are considered. The mathematical problem is reduced to a fourth-order, non-linear ordinary differential equation for a normalized, dimensionless temperature field. The nonlinearity enters the problem via the mean velocity, which is a parameter to 
be determined together with the solution. Global energy conservation yields an additional condition, which is used to solve for the mean velocity. The most striking finding of this work is the existence of different flow conditions, yielding either no solution, a unique solution, multiple or infinite solutions.

Several features of the flow and temperature fields which are derived directly by careful inspection of the governing equations and boundary conditions are briefly recalled:

- The dimensionless temperature $\Theta$ depends only on the dimensionless group $A \equiv \cos (\varphi) /\left(\mathrm{Ra}_{*} \sin ^{2} \varphi\right)$ and on the wall-normal coordinate $Y$. The channel inclination does not appear as an additional, independent dimensionless group affecting the dimensionless temperature.

- The dimensionless velocity $U$ depends on the dimensionless group $A$, on the channel inclination through the multiplicative factor $\sin (\varphi)$ and on the wall-normal coordinate $Y$. Under the additional assumptions of a nearly vertical channel or large $\mathrm{Ra}_{*} U_{m}$ product, it is shown that the dimensionless velocity does not depend on $A$, see equation (27a).

- The static pressure varies quadratically along the streamwise direction for the case of opposing mixed convection between inclined, parallel plates. The temperature field varies linearly along $x$.

Depending on the value assumed by the dimensionless parameter $A$, the following cases are discussed:

- Stably-stratified flow, $A>0$ (or $0<\varphi<\pi / 2$ ), $U_{m}>0$ : for $A>$ $3.25 \times 10^{-7}$ a unique solution exists, not displaying flow reversal. For $A \leqslant 3.25 \times 10^{-7}$, additional solutions appear by two at a time as $A$ decreases. The first order solution, corresponding to the largest value of $U_{m}$, does not show any flow reversal, while the higher order solutions show a progressively-increasing number of flow reversals, located near the bottom, adiabatic wall and extending towards the heated wall as the solution order increases.

- Stably-stratified flow, $A>0$ (or $0<\varphi<\pi / 2$ ), $U_{m}<0$ : this problem has infinitely many solutions for every given parameter $A$. Solutions of any order show flow reversal. The number of flow reversals increases progressively with increasing order of the solution. 
- Unstably-stratified flow, $A<0$ (or $\pi / 2<\varphi<\pi$ ), $U_{m}>0$ : for $|A|>1.14 \times 10^{-3}$ no solution exists. For $|A| \leq 1.14 \times 10^{-3}$, additional solutions appear in couples as $|A|$ decreases.

- Unstably-stratified flow, $A<0$ (or $\pi / 2<\varphi<\pi$ ), $U_{m}<0$ : this problem has infinitely many solutions for any given parameter $A$.

The mechanism leading to flow inversion is investigated. It is shown that the wall-normal variation of the streamwise pressure gradient is responsible for flow inversion, for large values of the parameter $A$.

The local Nusselt number, based on the difference between the top-walland the bulk-temperatures, is shown to be independent of the streamwise coordinate while depending only on the Rayleigh number and on the channel inclination through the single dimensionless group $A$.

Two-dimensional numerical simulations of developing flow and heat transfer are used to identify the physical solution in cases where multiple or infinite analytical solutions exist, for $U_{m}>0$ and $A>0$. The far-field numerical solution falls on the lowest order analytical solution corresponding to $\alpha_{0}$, irrespective of the initial conditions employed. Lavine [12] has already proved that the laminar solutions corresponding to $U_{m}<0$ are unstable, for any arbitrary value of $A$. The present numerical simulations suggest that the lower order solutions for $U_{m}>0$ might be the only stable solutions. A linear stability analysis could help clarifying this issue.

\section{A. Solution for positive mean velocity and $m=0$}

Enforcement of boundary conditions at $Y=0$ on equations (35) yield

$$
b=-a+A \alpha^{3}(\alpha-2) ; \quad c=a ; \quad d=a+A \alpha^{3}(\alpha+2)
$$

and

$$
\begin{aligned}
\Theta(Y) & =2 a \sin (\alpha Y) \cosh (\alpha Y) \\
& -2 \cos (\alpha Y) \sinh (\alpha Y)\left(a+2 A \alpha^{3}\right) \\
& +2 A \alpha^{4} \cos (\alpha Y) \cosh (\alpha Y) \\
& +4 A \alpha^{4}\left(Y-\frac{1}{2}\right)
\end{aligned}
$$


The constants $a$ and $\alpha$ have to be determined by imposing boundary conditions on the top wall. Imposing $\Theta^{\prime \prime}(1)=0$ yields

$$
\begin{aligned}
0 & =a \alpha^{2}[\cos (\alpha) \sinh (\alpha)+\cosh (\alpha) \sin (\alpha)] \\
& +A \alpha^{5} \sin (\alpha)(2 \cosh (\alpha)-\alpha \sinh (\alpha))
\end{aligned}
$$

while $\Theta^{\prime}(1)=1$ leads to

$$
\begin{aligned}
1 & =4 A \alpha^{4}(1-\cos (\alpha) \cosh (\alpha)) \\
& +4 \alpha\left(a+A \alpha^{3}\right) \sin (\alpha) \sinh (\alpha) \\
& +2 A \alpha^{5}(\cos (\alpha) \sinh (\alpha)-\sin (\alpha) \cosh (\alpha))
\end{aligned}
$$

The unknown $a$ can be expressed as function of $\alpha$ directly form equation (62)

$$
a=-\frac{A \alpha^{3} \sin (\alpha)(2 \cosh (\alpha)-\alpha \sinh (\alpha))}{D(\alpha)}
$$

provided the denominator $D(\alpha)$

$$
D(\alpha)=\cos (\alpha) \sinh (\alpha)+\cosh (\alpha) \sin (\alpha)
$$

is not equal to zero. As equation $(62)$ for $D(\alpha)=0$ becomes

$$
0=\sin (\alpha)(2 \cosh (\alpha)-\alpha \sinh (\alpha))
$$

it can be recognized that equation (66) and $D(\alpha)=0$ do not have solutions in common. Thus we can conclude that expression (64) does not alter the number of solutions to the problem.

Substitution of equation (64) into equation (63) results in an expression which provides $\alpha$ as a function of the parameter $A$ and has the following form

$$
f(\alpha)=\frac{1}{A}
$$

where

$$
f(\alpha) \equiv\left(\frac{\alpha \cos (2 \alpha)-2(\sin (2 \alpha)+\sinh (2 \alpha)+\alpha)+\alpha \cosh (2 \alpha)}{D(\alpha)}+4\right) \alpha^{4}
$$




\begin{tabular}{ccc}
\hline$k$ & $\alpha_{k}^{D}$ & $(k+3 / 4) \pi$ \\
\hline 0 & 2.365020372431352 & 2.356194490192345 \\
1 & 5.497803919000836 & 5.497787143782138 \\
2 & 8.639379828699742 & 8.639379797371930 \\
3 & 11.780972451020228 & 11.780972450961723 \\
4 & 14.922565104551627 & 14.922565104551516 \\
5 & 18.064157758141313 & 18.064157758141310 \\
\hline
\end{tabular}

Table 4: First 5 coefficients $\alpha_{k}^{D}$ and comparison with $(k+3 / 4) \pi$.

Function $D(\alpha)$ has an infinite sequence of roots identified as $\alpha_{k}^{D}$ for $k=$ $0,1 \ldots$ Few $\alpha_{k}^{D}$ values are provided in table 4 . The function $D(\alpha)$ is positive for $0<\alpha<\alpha_{0}^{D}$, it then becomes negative for $\alpha_{0}^{D}<\alpha<\alpha_{1}^{D}$ and has alternating sign over intervals of lengths approaching $\pi$ for $k \rightarrow \infty$. The roots of $D(\alpha)$ approach $(k+3 / 4) \pi$ for increasing $k$ (see table 4 ). The numerator of function $f(\alpha)$ instead is positive for $\alpha>\alpha_{0}^{D}$ and increasing like $(\alpha-$ 2) $\exp (2 \alpha)$.

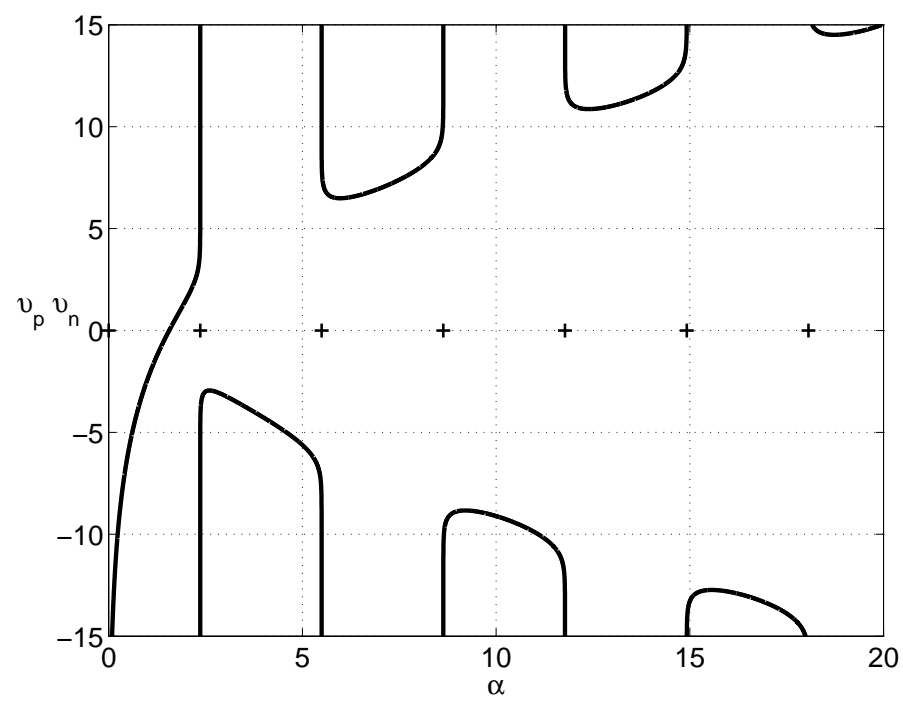

Figure 13: Graphs of $v_{p}$ and $v_{n}$. Plus symbols indicate $\alpha_{k}^{D}$, the first zeros of $D(\alpha)$.

To provide information on the behavior of function $f$, figure 13 shows the graphs of $v_{p}=\log _{10}(f)$ in $\left(\alpha_{2 q-1}^{D}, \alpha_{2 q}^{D}\right)$ and $v_{n}=-\log _{10}(-f)$ in $\left(\alpha_{2 q}^{D}, \alpha_{2 q+1}^{D}\right)$ 
for $q=1,2, \ldots$ Function $f$ approaches zero for $\alpha \rightarrow 0^{+}$and increases monotonically towards infinity for $\alpha \rightarrow \alpha_{0}^{-}$therefore between $0<\alpha<\alpha_{0}^{D}$ there are no $A<0$ solutions to equation (67) but there is always one solution for positive $A$. Function $f$ is positive and convex for $\left(\alpha_{2 q-1}^{D}, \alpha_{2 q}^{D}\right)$ while it is negative and concave in $\left(\alpha_{2 q}^{D}, \alpha_{2 q+1}^{D}\right)$ for $q=1,2 \ldots$, it goes to $\pm \infty$ for $\alpha$ approaching the $\alpha_{k}^{D}$ roots of $D(\alpha)$. Setting $A>0$ and therefore $\varphi<\pi / 2$, each interval $\left(\alpha_{2 q-1}, \alpha_{2 q}\right)$ for $q=1,2 \ldots$ adds two solutions to equation (67) provided $1 / A$ is larger than the local minimum of the function. In general for every given $A>0$ the number of solutions to problem (31) is

$$
n_{s}=1+2 n_{o}
$$

where $n_{o}$ is such that the minimum inside $\left(\alpha_{2 n_{o}-1}, \alpha_{2 n_{o}}\right)$ is smaller than $1 / A$, but this does not happen for $n_{o}+1$.

For generic $A$, the $n$-th solution $n=1, \ldots n_{s}$ is obtained by substitution of expression (64) calculated for $\alpha_{n}$ in the generic temperature solution (61). After the temperature profile is known, velocity profiles can be obtained from the second derivative of $\Theta$, through equation (23c).

\section{B. Solution for negative mean velocity and $m=0$}

The boundary conditions at $Y=0$ applied to equation (35) yield the following equations

$$
b=\frac{A \sigma^{4}}{4}-a ; \quad c=2 a-A \sigma^{3}-\frac{A \sigma^{4}}{4} ; \quad d=\frac{A \sigma^{4}}{4}
$$

and

$$
\begin{aligned}
\Theta(Y) & =\frac{A}{4}\left[\sigma^{4} e^{\sigma y}-\left(\sigma^{4}+4 \sigma^{3}\right) \sin (\sigma y)+\sigma^{4} \cos (\sigma y)+4 \sigma^{4}\left(y-\frac{1}{2}\right)\right] \\
& -2 a[\sinh (\sigma y)-\sin (\sigma y)]
\end{aligned}
$$

The boundary condition $\Theta^{\prime \prime}(1)=0$ yields

$$
\begin{aligned}
0 & =\frac{A}{4}\left[\left(e^{\sigma}+\sin (\sigma)-\cos (\sigma)\right) \sigma^{6}+4 \sin (\sigma) \sigma^{5}\right] \\
& -2 a \sigma^{2}(\sinh (\sigma)+\sin (\sigma))
\end{aligned}
$$


while from $\Theta^{\prime}(1)=1$ it results

$$
\begin{aligned}
1 & =\frac{A}{4}\left[\left(e^{\sigma}-\cos (\sigma)-\sin (\sigma)\right) \sigma^{5}+4(1-\cos (\sigma)) \sigma^{4}\right] \\
& +a \sigma\left[\left(2 \cos (\sigma)-e^{-\sigma}-e^{\sigma}\right)\right]
\end{aligned}
$$

The constant $a$ can be expressed as function of $\sigma$ from (72):

$$
a=\frac{A\left[\left(e^{\sigma}+\sin (\sigma)-\cos (\sigma)\right) \sigma^{4}+4 \sin (\sigma) \sigma^{3}\right]}{8(\sinh (\sigma)+\sin (\sigma))}
$$

expression (74) is always valid as $\sigma>0$.

An expression for the temperature field in terms of the parameter $A$ and the constant to be determined $\sigma$ can be recovered from equations (71) and (74). The following equation for $\sigma(A)$

$$
\begin{aligned}
0 & =\frac{A}{4}\left\{\left[\left(-e^{\sigma}+\cos (\sigma)+\sin (\sigma)\right)(\sinh (\sigma)+\sin (\sigma))\right.\right. \\
& \left.+\left(e^{\sigma}+\sin (\sigma)-\cos (\sigma)\right)(\cosh (\sigma)-\cos (\sigma))\right] \sigma^{5}+ \\
& \left.+4[\cos (\sigma) \sinh (\sigma)-\sinh (\sigma)-\sin (\sigma)+\sin (\sigma) \cosh (\sigma)] \sigma^{4}\right\}+ \\
& +[\sinh (\sigma)+\sin (\sigma)]
\end{aligned}
$$

is obtained by subtraction of equation (73) times $\sinh (\sigma)+\sin (\sigma)$ from $(72)$ times $\cosh (\sigma)-\cos (\sigma)$. This operation does not add any root to equations (72) and (73) because $\sinh (\sigma)+\sin (\sigma)$ and $\cosh (\sigma)-\cos (\sigma)$ do not vanish for $\sigma>0$.

An expression of the form

$$
g(\sigma)=\frac{1}{A}
$$

can be easily obtained by dividing each side of equation (75) by $A(\sinh (\sigma)+\sin (\sigma))$. The study of equation (76) reveals that for $U_{m}<0$ this problem has infinitely many solutions for every given parameter $A$. The first-order root $\sigma_{1}$ is provided for different $A$ values in table (5). 


\begin{tabular}{rr}
\hline $\log (A)$ & $\sigma_{1}(A)$ \\
\hline-5 & 11.786354443548641 \\
-4 & 10.715999778187648 \\
-3 & 4.729310587146569 \\
-2 & 3.586967463328612 \\
-1 & 2.840255248497744 \\
0 & 2.296896837286697 \\
1 & 1.877383319551463 \\
2 & 1.542462128734405 \\
3 & 1.270410029398276 \\
5 & 0.864247016407518 \\
10 & 0.330977981272878 \\
15 & 0.126802797233359 \\
\hline
\end{tabular}

Table 5: Values of $\sigma_{1}(A)$ for a wide range of values for the parameter $A$.

For $\sigma \gtrsim 3 \pi / 2$ function $g(\sigma)$ is well approximated by $\widetilde{g}$

$$
\widetilde{g}(\sigma)=\frac{1}{2}\left[\cos (\sigma) \sigma^{5}-2(\cos (\sigma)+\sin (\sigma)-1) \sigma^{4}\right]
$$

and the graphs of both functions $g$ and $\widetilde{g}$ are provided in figure 14. This reveals that infinite many solutions to equation (75) are found in the range $2^{\frac{1}{5}} A^{-\frac{1}{5}}<\sigma<\infty$, that solutions are rather independent of $A$ and fall about $\sigma_{k} \approx\left(k+\frac{1}{2}\right) \pi$ for $k=2,3, \ldots$ Conversely the smallest $\sigma(A)$ root, indicated by $\sigma_{1}$ shows a dependence on $A$; sample $\sigma_{1}$ values are given in table 5 .

\section{References}

[1] Akbari, H., Borges, T., 1979. Free convective laminar flow within the trombe wall channel. Sol. Energy 22, 165-174.

[2] Aung, W., Kessler, T., Beitin, K., 1973. Free convection cooling of electronic systems. IEEE Trans. Parts, Hybrids Packag. PHP-9, 75-86.

[3] Batchelor, G., 1967. An introduction to fluid mechanics. Cambridge University Press, New York (NY), USA. 


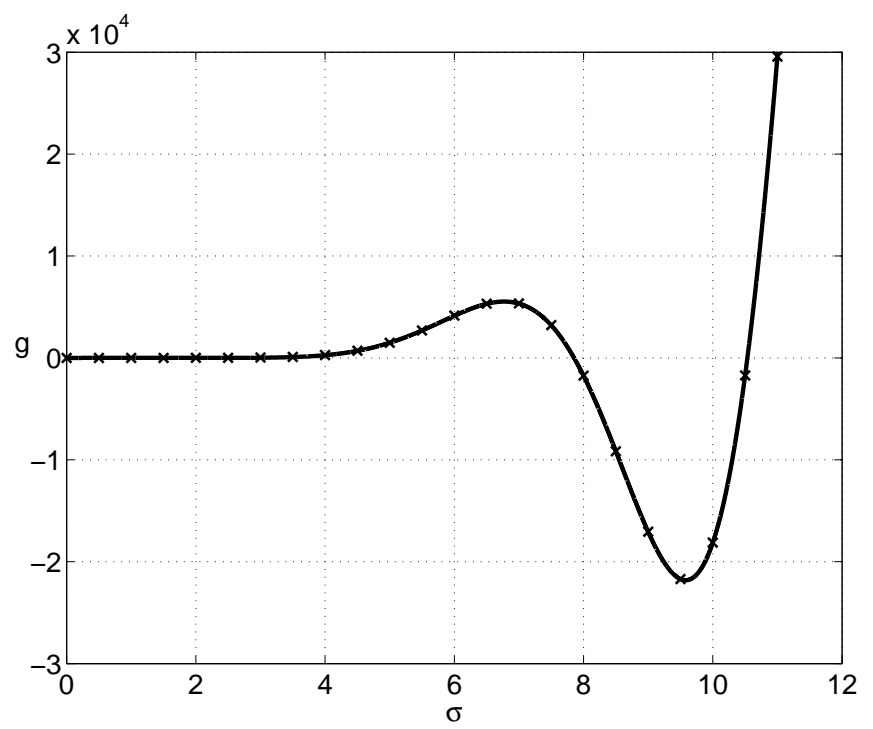

Figure 14: Graph of function $g(\sigma)$ for $\sigma \in[0,12]$; x-symbols display the approximation $\widetilde{g}$.

[4] Bergholz, R., 1978. Instability of steady natural convection in a vertical fluid layer. Journal of Fluid Mechanics 84, 743-768.

[5] Gelfgat, A., Bar-Yoseph, P., Yarin, A., 1999. Stability of multiple steady states of convection in laterally heated cavities. Journal of Fluid Mechanics $388,315-334$.

[6] Gershuni, G., Zhukovitskii, E., 1976. Convective Stability of Incompressible Fluids. Keter Publications, Jerusalem/Wiley.

[7] Hart, J., 5 1971. Stability of the flow in a differentially heated inclined box. Journal of Fluid Mechanics 47, 547-576.

[8] Inasaki, T., Komori, K., 1995. Numerical modeling of turbulent transport with combined forced and natural convection between two vertical parallel plates. Int. J. Comput. Meth. A 27, 417-431.

[9] Incropera, F., DeWitt, D., Bergman, T., Lavine, A., 2007. Fundamentals of Heat and Mass Transfer, 5th Edition. John Wiley \& Sons, Inc., Hoboken (NJ), USA.

[10] Lavine, A., July 1988. Analysis of fully developed aiding mixed convection between inclined parallel plates. In: ASME Proceedings of the 
1988 National Heat Transfer Conference. Vol. 96. American Society of Mechanical Engineers, Heat Transfer Division, Houston, TX, USA, pp. 87-94.

[11] Lavine, A., 1988. Analysis of fully developed opposing mixed convection between inclined parallel plates. Wärme- und Stoffübertragung 23, 249257.

[12] Lavine, A., 1993. On the linear stability of mixed and free convection between inclined parallel plates with fixed heat flux boundary conditions. Int. J. Heat Mass Transfer 36 (5), 1373-1381.

[13] Lin, C. C., 1944. On the stability of two-dimensional parallel flows. Proc Natl Acad Sci USA 30 (10), 316-324.

[14] Ostrach, S., 1952. Laminar natural-convection flow and heat transfer of fluids with and without heat sources in channels with constant wall temperatures. Technical note 2863, National Advisory Committee for Aeronautics, Lewis Flight Propulsion Laboratory, Cleveland, Ohio, USA, december.

[15] Piller, M., Stalio, E., 2012. Numerical investigation of natural convection in inclined parallel-plate channels partly filled with metal foams. Int. J. Heat Mass Transfer 55, 6506-6513.

[16] Salsa, S., 2008. Partial differential equations in action. Springer-Verlag, Milano, ITALY.

[17] Sparrow, E., Azevedo, L., 1985. Vertical-channel natural convection spanning between the fully-developed limit and the single-plate boundary-layer limit. Int. J. Heat Mass Transfer 28, 1847-1857.

[18] Vest, C., Arpaci, V., 1969. Stability of natural convection in a vertical slot. Journal of Fluid Mechanics 36 (1), 1-15.

[19] Webb, B., Hill, D., 1989. High rayleigh number laminar natural convection in an asymmetrically heated vertical channel. Journal of Heat Transfer, Transactions ASME 111 (3), 649-656. 